\title{
Differentiation of Fresh and Processed Fruit Juices Using Volatile Composition
}

\author{
Rosa Perestrelo $^{1, *}$, Catarina Silva ${ }^{1}\left(\mathbb{C}\right.$, Pedro Silva $^{1}\left(\mathbb{D}\right.$, Sonia Medina ${ }^{1}\left(\mathbb{C}\right.$ and José S. Câmara ${ }^{1,2, *}$ \\ 1 CQM-Centro de Química da Madeira, Universidade da Madeira, Campus da Penteada, 9020-105 Funchal, \\ Portugal; cgsluis@uma.pt (C.S.); pedro_dasilva@hotmail.com (P.S.); sonia.escudero@staff.uma.pt (S.M.) \\ 2 Departamento de Química, Faculdade de Ciências Exatas e Engenharia, Universidade da Madeira; \\ Campus da Penteada, 9020-105 Funchal, Portugal \\ * Correspondence: rmp@uma.pt (R.P.); jsc@uma.pt (J.S.C.); Tel.: +351-291705119 (R.P.); +351-291705112 (J.S.C.)
}

Received: 15 January 2019; Accepted: 6 March 2019; Published: 10 March 2019

\begin{abstract}
In the current study, a comprehensive approach based on headspace solid-phase microextraction (HS-SPME), combined with gas chromatography-quadrupole mass spectrometry (GC-qMS), was used to establish the volatile signature of fresh and processed fruit juices, obtained from the same batch of grapes, red fruits, orange, pear, and apple. This is a powerful tool for evaluating the impact of the production process on the volatomic pattern of fruit juice. A total of 169 volatile organic compounds (VOCs) belonging to different chemical groups were identified. Esters, carbonyl compounds, terpenoids, and alcohols are the major chemical groups in the investigated fruit juices. However, their contribution to the total volatile profile varied. Special attention should be paid to processed fruit juices to avoid the possible deleterious effects associated with the formation of furanic compounds (e.g., heat treatment), since their furanic content was significantly higher in comparison to that of fresh fruit juices. The knowledge obtained in the current study will allow for the introduction of modifications to the process involved in processing juice, which will improve the organoleptic characteristics of processed juices, contributing to a better acceptance by consumers. Furthermore, more assays should be performed to assess the effect of harvests, geography, and agronomy on the volatile profile of juices.
\end{abstract}

Keywords: volatile pattern; fresh juice; processed juice; HS-SPME; GC-MS

\section{Introduction}

A balanced diet that includes fruit benefits health. Fruit consumption may contribute to interference with cancer mechanisms, assist in ameliorating mutagenic, inflammatory, and neurodegenerative mechanisms, as well as contribute to some antimicrobial effects [1,2]. It represents a rich source of vitamins (mainly vitamin C), pectin, fibers, organic acids, and secondary metabolites originating predominantly from plant metabolism, including volatile organic compounds (VOCs), phenolic compounds, etc. [3,4]. Furthermore, most of the fruits are used in many foodstuffs, such as jams, liquors, wine, dairy products, and breakfast cereals [3].

The volatile profile of fruits is responsible for their aroma and is constituted by a complex mixture of hundreds of VOCs belonging to different chemical groups. These VOCs are dominated by four biosynthetic classes: terpenoids, fatty acid derivatives, compounds with aromatic rings (derived from L-phenylalanine), and volatiles derived from amino acid metabolism (methyl-branched alcohols, ketones, esters, sulfur containing and aromatic compounds) [5,6]. More than 300 VOCs have been identified in the aroma profile of apples [5], esters being the most dominant chemical group detected in different apple cultivars. From this chemical group, 2-methyl butyl acetate, hexyl acetate, butyl acetate, 2-methyl butanoate, ethyl butanoate, and ethyl methyl propanoate were the 
most important esters contributing to the characteristic apple aroma [6-9]. In pear fruit, esters were also the most significant contributors to aroma [10], being methyl and hexyl esters of decadienoate, the character imparting compounds of the European pear [11]. Other esters, such as hexyl acetate, 2-methylpropyl acetate, butyl acetate, and pentyl acetate, as well as butyl butanoate, ethyl hexanoate possessed strong pear-like odors [12]. In addition, hexanal, 2-methylpropyl acetate, ethyl acetate, hexyl acetate, 3-methylbutyl-2-methyl butanoate, ethyl butanoate, and butanol were identified as impact volatiles in Conference pears (Pyrus communis L.) [13]. Orange is a large cultivated fruit and studied worldwide. A total of 58 VOCs, including esters, terpenoids, carbonyl compounds, alcohols, and acids were identified and quantified in Dortyol yerli orange juice. Terpenoids were the most representative chemical group, limonene being the predominant VOC, followed by valencene, linalool, terpinen-4-ol, and $\alpha$-terpineol [14]. On the other hand, grapes (Vitis vinifera L.) volatiles include a great number of VOCs, namely monoterpenoids, sesquiterpenoids, carbonyl compounds, alcohols, $\mathrm{C}_{13}$ norisoprenoids, and acids [15]. Linalool, geraniol, citronellol, nerol, dienol I and II were the most abundant terpenoids in Muscat grape [16]. Other monoterpenes potentially contributing to Muscat aroma were rose oxide, citral, geraniol, nerol, and citronellol [17]. The evolution of terpenic compounds was evaluated during ripening of four V. vinifera L. grape varieties, Bual, Malvasia, Sercial (white grapes), and Tinta Negra (red grapes). Linalool, citronellol, geraniol, $\alpha$-ylangene, bicyclogermacrene, $\beta$-ciclocitral, $\beta$-damascenone are common to all grape varieties studied and were detected in all ripening stages, whereas others were found only in some ripening stages and in some varieties (e.g., $\beta$-ocimene isomer, $\beta$-gurjunene, $\gamma$-elemene). In addition, for all $V$. vinifera $L$. grapes studied, the maximum potential of mono-, sesquiterpenic, and norisoprenoid compounds was achieved at maturity date, which was established based on the maximum sugar content and minimum titratable acidity [18]. Even at low levels, these VOCs may have a significant impact on the overall flavor of fruits and their corresponding juices due to their lower odor threshold (OT). The levels of these VOCs are frequently low (typically $\mu \mathrm{g} / \mathrm{L}$ ) and are influenced by a number of agronomic (e.g., variety, climatological conditions, ripening stage) [19] and technological factors (e.g., harvest, post-harvest treatments, storage and processing conditions) [20]. During fruit juice processing, the volatile signature is affected by the addition of additives, preservatives, and chemical or processing treatments that change the volatile profile of fresh juices [21].

Temperatures between 85 and $95{ }^{\circ} \mathrm{C}$ are frequently used to extend the shelf life of fruit juices However, it has been documented that the thermal process may activate the dormant ascospores of molds, which subsequently cause deterioration, hence resulting in economic loss [22]. Heat treatment with high temperature and long processing time is not recommended, due to quality reasons and consumer demands for fresh-like fruits. To overcome this problem on fruit juice preservation, non-thermal methods, such as high-pressure processing (HPP), supercritical carbon dioxide technology (SCCD), and power ultrasound in combination with mild heat have been proposed. Yi et al. [23] evaluated the impact of HPP (600 MPa, $3 \mathrm{~min}$ ) on apple (Pink Lady, Granny Smith, and Jonagold) juice quality changes and compared it to the thermal processing. The overall quality of HPP treated apple juices was much more comparable to that of the fresh juice, in particular HPP results in lower amounts of thermally induced compounds that are related to cooked notes of pasteurized apple juices. Kebede et al. [24] investigated the volatile changes during the shelf life of pulsed electric fields (PEF) $(15.5 \mathrm{kV} / \mathrm{cm}$ and specific energy of $158 \mathrm{~kJ} / \mathrm{L}), \mathrm{HPP}(600 \mathrm{MPa}$ for $3 \mathrm{~min})$, and thermally $\left(72{ }^{\circ} \mathrm{C}\right.$ for $15 \mathrm{~s})$ pasteurized Jazz apple juices, up to five weeks. The results showed that after pasteurization, PEF processing resulted in a better retention of odor active VOCs, such as 2-hexenal isomer and hexyl acetate, whereas thermal processing lowered their amount. Gao et al. [25] evaluated the effects of $\mathrm{HPP}\left(550 \mathrm{MPa} / 10 \mathrm{~min}\right.$ ) and high-temperature short time (HTST; $110^{\circ} \mathrm{C} / 8.6 \mathrm{~s}$ ) on microorganisms, ascorbic acid, total phenols, antioxidant capacity, color, enzyme activity, and rheological behavior in red grapefruit juice during 30 days of storage at $4{ }^{\circ} \mathrm{C}$. Evelyn et al. [26] showed that HPP pretreatment enhanced the thermosonication inactivation of Alicyclobacillus acidoterrestris spores in orange juice, whereas Mastello et al. [27] evaluated the impact of HPP processing on the volatile profile and sensory 
acceptance of Pêra-Rio orange juice. Moreover, several studies have demonstrated the efficiency of SCCD processing in the preservation of juices such as orange, melon, kiwi, pear, and strawberry. The findings confirm the SCCD processing as a potential promising technology to the conventional thermal treatment [28-30]. The assessment of the volatile signature of fruit juice is of great importance, since it enables researchers to help producers improve its quality, develop new products for the market, contribute to its economic valorization [27], as well as to assess the geographical origin, authenticity, and typicity of food matrices [31].

Several extractions and enrichment techniques, namely, liquid-liquid extraction (LLE) [32], simultaneous distillation extraction (SDE) [33], solid-phase trapping solvent extraction (SPTE) [33], solid-phase extraction (SPE) [34], pressurized-fluid extraction (PFE) [35], solid-phase microextraction (SPME) [3,33], and stir bar sorptive extraction (SBSE) [36] have been applied to establish the volatile signature of food matrices. Nevertheless, microextraction techniques (METs) (e.g., SPME, SBSE) compared to conventional extraction techniques (e.g., LLE, SDE, SPE) offer several advantages, such as a reduction of the amount of organic solvent, the possibility of automation, high sensitivity and concentration factors [37]. These METs, combined with analytical measurements, can be used to assess the sensory properties, chemical composition, authenticity, and typicality of juices. The establishment of the volatile signature, analysis of the nutritional composition, phenolic compounds, carotenoids, and some vitamins [3,38], in addition to sensory analysis using consumers and/or a trained taste panel, or even using an instrumental taste sensing system, like the electronic tongue [39], are the most widely used techniques to assess the quality of fruit juices [40].

The main goal of this study was to establish and compare the volatile signature of fresh and processed fruit juices, obtained from the same batch of fruits, by means of headspace solid-phase microextraction (HS-SPME), combined with gas chromatography-quadrupole mass spectrometry (GC-qMS). In addition, the impact of processing on the volatile signature of juices from grapes (Vitis vinifera L.), wild red fruits (Fragaria $\mathrm{x}$ ananassa Duch., Vaccinium corymbosum L., Rubus idaeus L.), orange (Citrus sinensis L.), pear (Pyrus communis L.), and apple (Malus domestica Borkh.), as well as the corresponding processed juices (from the same batch), was investigated. These comprehensive data will allow the evaluation of the impact of processing on the volatile signature of fruit juices from the same batch. As far as we know, this is the first time that different fruit juices corresponding to different fruit species were analyzed simultaneously using the same analytical technique, therefore enabling us to describe both the volatile profile in the juice and the variability in the volatile profile between fresh and processed fruit juices. The obtained results may help to enlarge the database on the volatile and aroma composition of fresh and processed fruit juices, as well as provide a useful platform to improve the organoleptic characteristics of the processed juices and consequently contribute to their economic valorization and improve their acceptance by consumers.

\section{Results and Discussion}

\subsection{Volatomic Signature of Fresh and Processed Fruit Juices}

A comprehensive untargeted analysis of the volatile signature of fresh and processed fruit juices, obtained from the same batch of fruits, was performed using HS-SPME/GC-qMS. The data were processed using software (NIST, 2005; Mass Spectral Search Program V.2.0d, Agilent, Washington, USA), that provides quality matching using advanced spectral matching algorithms background subtraction and kovats index (KI) comparison. Figure 1 shows a typical total ion chromatogram (TIC) of fresh (top) and processed (bottom) pear juices obtained by HS-SPME/GC-qMS. Great differences among the patterns of the VOCs from fresh and processed pear juices were observed. On average, 125 VOCs were identified in the headspace of the investigated processed fruit juices (108 in grape, 117 in red fruits, 97 in orange, 123 in pear, and 124 in apple juice), whereas the volatile signature of fresh juices was less complex, with 111 VOCs identified (84 in grape juice, 111 in red fruits, 98 in orange, 108 in pear, and 109 in apple juices) (Figure S1, Supplementary Materials). The identified VOCs 
belonged to distinct chemical groups, including terpenoids (57), esters (56), carbonyl compounds (33), alcohols (16), acids (3), volatile phenols (2), and furanic compounds (2) (Table 1). Moreover, some differences were also observed in both the qualitative and semi-quantitative (GC peak area) expressions. Table 1 shows the KI determined with a BP20 column, as well as the KI reported in the scientific literature with equivalent columns, was used to confirm the identity of VOCs. Thirty-six of the identified VOCs were common to all the fresh and processed juices investigated (Table S1, Supplementary Materials). On the other hand, some VOCs were detected in specific juices, such as 3-methylbutanal in grape juice, hexyl butanoate and rose oxide isomer in red fruit juices, p-cymene, safranal, and carvone in orange juice, ethyl pentanoate and geranyl valerate in apple juice, and geranial in pear juice.

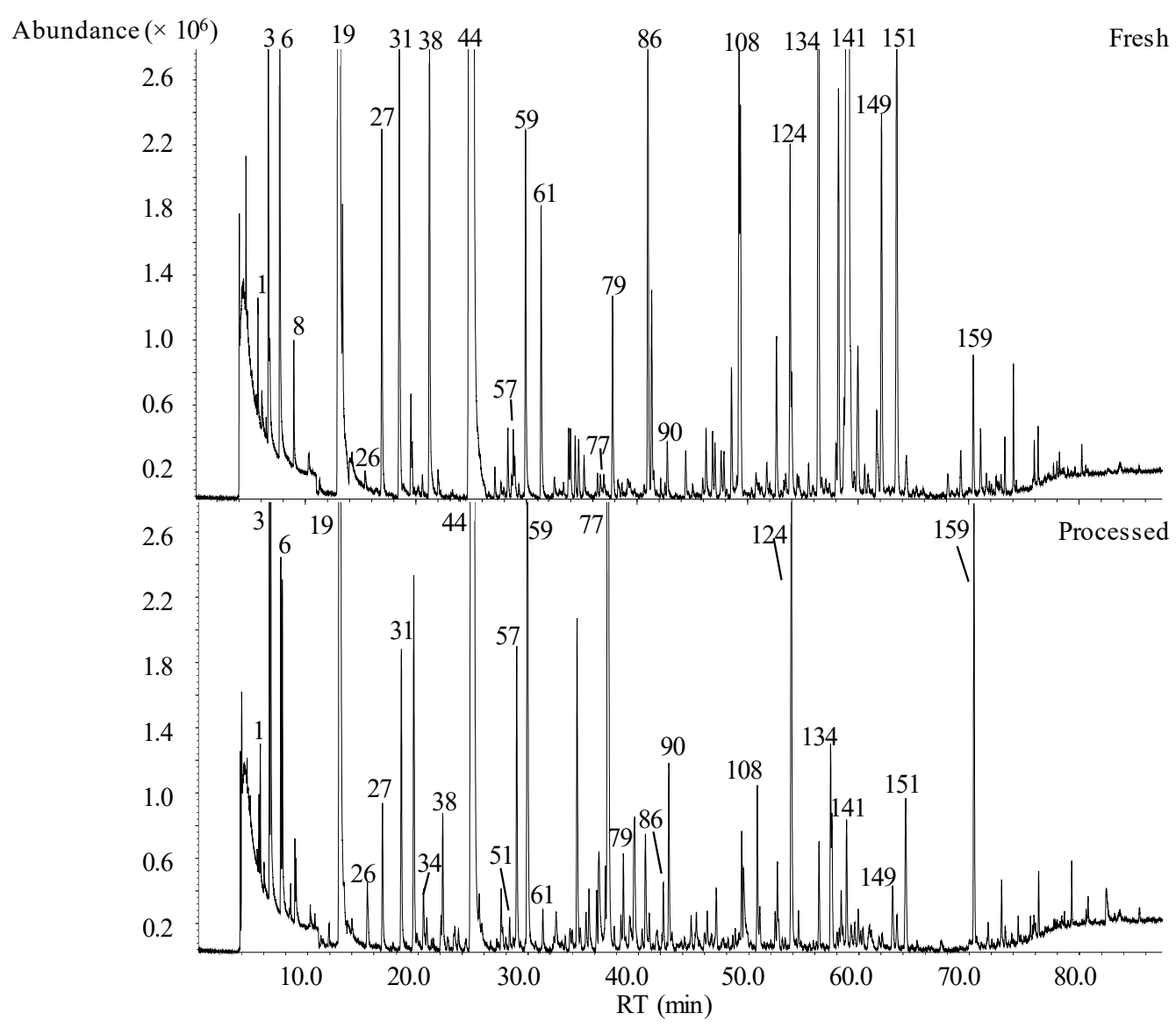

Figure 1. Total ion chromatograms obtained by the headspace solid-phase microextraction

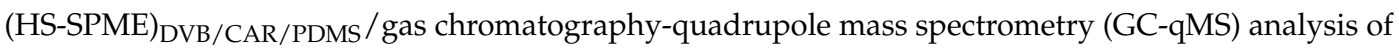
fresh and processed pear juice (for the identification of peak numbers, see Table 1).

The distribution of the chemical groups, according to the production process of juices, is shown in Figure 2. Esters, carbonyl compounds, terpenoids, and alcohols are the predominant chemical groups detected in fruit juices. However, significant differences were observed among these chemical groups, according to the production process and fruit variety. The esters were the predominant chemical group in all of the investigated fruit juices (55.2\%, on average), except for orange and grape juices, which account for 23.6 and $26.0 \%$ of the total volatile profile of the fresh juices, and 20.9 and $18.5 \%$ of that of processed juices, respectively. However, the GC total peak areas of esters were, on average, 1.1 and 1.4 times higher in fresh apple juices than the corresponding processed juices. Hexyl acetate was the most abundant ester identified in red fruits, pear, and apple juices, irrespective of the type of juice (Table 1 and Table S2). 
Table 1. GC peak area $\left(\times 10^{6}\right)$ of volatile organic compounds (VOCs) identified in fresh and processed fruit juices.

\begin{tabular}{|c|c|c|c|c|c|c|c|c|c|c|c|c|c|c|c|c|c|c|c|c|c|c|c|c|}
\hline \multirow{3}{*}{$\begin{array}{c}\text { Peak } \\
n^{\circ}\end{array}$} & \multirow{3}{*}{$\underset{(\min )^{1}}{\text { RT }}$} & \multirow{3}{*}{$\mathrm{KI}_{\mathrm{Cal}}{ }^{2}$} & \multirow{3}{*}{$\mathrm{KI}_{\mathrm{Lit}}{ }^{3}$} & \multirow{3}{*}{ Chemical Groups } & \multicolumn{20}{|c|}{ GC Peak Area $\left(\times 10^{6}\right)($ RSD) } \\
\hline & & & & & \multicolumn{4}{|c|}{ Grape } & \multicolumn{4}{|c|}{ Red Fruits } & \multicolumn{4}{|c|}{ Orange } & \multicolumn{4}{|c|}{ Pear } & \multicolumn{4}{|c|}{ Apple } \\
\hline & & & & & \multicolumn{2}{|c|}{ Fresh } & \multicolumn{2}{|c|}{ Processed } & \multicolumn{2}{|c|}{ Fresh } & Proces & ssed & Fre & & Proce & ssed & Fres & & Proces & ssed & Fres & & Proce & essed \\
\hline & & & & Esters & & & & & & & & & & & & & & & & & & & & \\
\hline 1 & 5.67 & 897 & 864 & Methyl acetate & 11.18 & (2) & 2.17 & (5) & 29.89 & (7) & 16.76 & $(8)$ & 1.23 & (2) & 1.98 & (5) & 23.94 & (16) & 21.71 & (13) & 1.98 & (19) & 2.58 & (8) \\
\hline 3 & 6.59 & 925 & 907 & Ethyl acetate ${ }^{4}$ & 51.31 & (3) & 12.45 & (5) & 4.58 & (6) & 14.56 & (15) & 7.83 & (11) & 3.08 & (15) & 222.09 & (13) & 257.16 & (10) & 144.12 & (8) & 131.55 & (16) \\
\hline 7 & 8.38 & 969 & 950 & Ethyl propanoate ${ }^{4}$ & 7.97 & (6) & 26.03 & (5) & 10.21 & (4) & 23.32 & (7) & - & & - & & - & & - & & 28.73 & (6) & 7.32 & (12) \\
\hline 8 & 8.95 & 981 & 969 & Propyl acetate 4 & 5.66 & (6) & 0.70 & (9) & & & & & & & - & & 9.82 & (5) & 35.95 & (1) & 0.76 & (18) & 17.32 & (1) \\
\hline 10 & 9.29 & 988 & 982 & Methyl butanoate & - & & - & & 94.87 & (12) & 46.71 & (8) & $1.27^{\mathrm{a}}$ & (3) & $1.45^{\mathrm{a}}$ & (1) & - & & - & & 6.14 & (4) & 1.51 & (13) \\
\hline 11 & 10.17 & 992 & 1007 & Methyl 2-methylbutanoate & 12.72 & (11) & 1.48 & (13) & 1.06 & (3) & - & & - & & - & & 2.15 & (3) & 4.52 & (16) & 10.32 & (9) & 12.34 & (5) \\
\hline 13 & 10.53 & 1002 & 1015 & Methyl 3-methylbutanoate & - & & - & & 13.65 & (3) & 17.86 & (6) & & & - & & 3.20 & (1) & 5.41 & (9) & & & & \\
\hline 15 & 11.33 & 1024 & 1028 & Ethyl butanoate ${ }^{4}$ & 17.95 & (6) & 6.42 & (3) & 30.48 & (8) & 18.24 & (8) & 64.68 & (10) & 62.50 & (5) & - & & - & & 10.08 & (6) & 1.94 & (8) \\
\hline 16 & 12.08 & 1043 & 1050 & Ethyl 2-methylbutanoate & - & & - & & 1.72 & (18) & 42.60 & (5) & 2.35 & (12) & 1.94 & (9) & - & & - & & 21.85 & (9) & 2.19 & (15) \\
\hline 18 & 12.75 & 1059 & 1060 & Ethyl 3-methylbutanoate & - & & 10.39 & (4) & 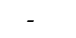 & & & & 1.25 & (4) & 2.16 & (6) & - & & 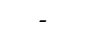 & & & & 40.51 & (2) \\
\hline 19 & 13.02 & 1065 & 1075 & Butyl acetate ${ }^{4}$ & 2.18 & (11) & 2.34 & (17) & 67.76 & (2) & 56.12 & (4) & - & & - & & 21.32 & (4) & 16.93 & (6) & 132.24 & (13) & 221.32 & (8) \\
\hline 22 & 15.40 & 1115 & 1117 & 3-Methylbutanol acetate 4 & 10.03 & (13) & 0.88 & (11) & 19.70 & (7) & 5.20 & (15) & - & & - & & 6.55 & (12) & 19.26 & (14) & 617.98 & (12) & 745.10 & (8) \\
\hline 25 & 16.18 & 1130 & 1120 & Ethyl pentanoate & - & & - & & - & & & & - & & - & & - & & - & & 6.03 & (13) & 11.65 & (5) \\
\hline 26 & 16.51 & 1136 & 1120 & Butyl propanoate & - & & - & & 6.05 & (14) & 1.46 & (7) & 11.25 & (3) & 26.43 & (3) & 22.63 & (1) & 40.01 & (9) & 33.36 & (18) & 49.34 & (4) \\
\hline 28 & 17.65 & 1156 & 1160 & Propyl propanoate & - & & - & & 5.51 & (7) & 10.41 & (9) & 14.16 & (4) & 66.78 & (6) & 0.65 & (13) & 1.97 & (6) & 1.18 & (9) & 7.71 & (6) \\
\hline 30 & 17.88 & 1159 & 1152 & Ethyl 2-butenoate & 6.73 & (10) & 0.52 & (13) & & & & & 15.49 & (14) & 27.52 & (2) & - & & & & 3.26 & (3) & & \\
\hline 31 & 18.52 & 1170 & 1147 & Pentyl acetate & 7.25 & (8) & 0.78 & (11) & 2.78 & (8) & 5.89 & (3) & - & & - & & 16.30 & (9) & 2.02 & (9) & 13.85 & (14) & 1.90 & (6) \\
\hline 34 & 19.28 & 1182 & 1188 & Methyl hexanoate & - & & - & & 564.99 & (7) & 430.05 & (7) & - & & - & & - & & 158.81 & (3) & 13.23 & (8) & 28.72 & (9) \\
\hline 40 & 22.23 & 1229 & 1228 & Propyl butanoate & 4.59 & (6) & 0.74 & (15) & - & & - & & - & & - & & - & & 48.35 & (7) & - & & 10.56 & (15) \\
\hline 41 & 22.36 & 1231 & 1220 & Ethyl hexanoate ${ }^{4}$ & 27.68 & (11) & 1.19 & (5) & 77.27 & (13) & 82.60 & (15) & 23.42 & (7) & 52.65 & (15) & 1.82 & (16) & 6.00 & (9) & 34.09 & (7) & 2.50 & (1) \\
\hline 44 & 24.85 & 1269 & 1270 & Hexyl acetate ${ }^{4}$ & 31.63 & (4) & 12.66 & (12) & 356.35 & $(8)$ & 233.56 & (5) & 53.36 & (9) & 39.23 & (2) & 676.33 & (6) & 368.37 & (1) & 193.62 & (10) & 109.58 & (5) \\
\hline 49 & 26.85 & 1296 & 1292 & Ethyl 3-hexenoate ${ }^{4}$ & - & & - & & 3.06 & (12) & 1.28 & (2) & - & & - & & 2.09 & (14) & 4.47 & (5) & 1.01 & (14) & & \\
\hline 50 & 27.11 & 1300 & 1305 & Ethyl 2-hexenoate & 2.58 & (9) & 1.14 & (12) & 3.25 & (5) & 8.77 & (4) & - & & - & & 11.41 & (5) & 3.99 & (8) & 0.98 & (5) & 3.36 & (8) \\
\hline 51 & 27.51 & 1306 & 130 & Ethyl 3-ethoxypropanoate & - & & - & & - & & - & & - & & - & & - & & 22.33 & (5) & 4.02 & (15) & - & \\
\hline 52 & 27.76 & 1310 & 1328 & 3-Hexen-1-ol acetate isomer & 4.27 & (3) & 3.36 & (3) & 36.28 & (2) & 142.79 & (2) & - & & - & & 5.91 & (14) & 9.81 & (7) & 78.43 & (14) & 56.48 & (5) \\
\hline 54 & 28.31 & 1319 & 1327 & 2-Hexen-1-ol acetate isomer & 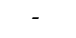 & & 0.25 & (12) & 5.57 & (7) & 4.12 & (1) & 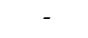 & & - & & 27.12 & (9) & 11.09 & (8) & 3.32 & (17) & 2.88 & (6) \\
\hline 56 & 29.10 & 1332 & 1332 & Hexyl propanoate & - & & 0.76 & (11) & 26.11 & (4) & 7.36 & (11) & - & & - & & 6.93 & (19) & 3.66 & (7) & 4.42 & (12) & 4.52 & (12) \\
\hline 57 & 29.37 & 1336 & 1305 & Ethyl 2-hexenoate & 3.12 & (5) & 8.58 & (13) & 0.39 & (6) & 2.53 & (4) & 2.01 & (5) & 5.47 & (8) & 1.52 & (17) & 2.24 & (7) & 6.87 & (16) & 3.75 & (1) \\
\hline 61 & 31.36 & 1366 & 1359 & Heptyl acetate & 5.91 & (12) & 0.33 & (9) & - & & - & & 12.28 & (8) & 23.95 & (7) & 20.09 & (14) & 6.38 & (4) & 1.26 & (7) & 30.99 & (3) \\
\hline 64 & 32.35 & 1380 & 138 & Methyl octanoate & - & & - & & 10.17 & (15) & 5.98 & (2) & & & - & & 0.93 & (13) & 1.76 & (15) & & & - & \\
\hline 68 & 33.66 & 1398 & 1391 & Butyl methylbutanoate & - & & - & & 21.27 & (12) & 7.13 & (1) & 2.61 & (3) & 1.33 & (2) & 21.82 & (4) & 9.65 & (11) & & & & \\
\hline 69 & 33.99 & 1404 & 1394 & Ethyl 2-hydroxyisovalerate & - & & - & & 36.49 & (10) & 10.24 & (2) & 2.66 & (17) & 2.25 & (6) & 29.38 & (12) & 7.58 & (18) & 7.22 & (13) & 3.23 & (6) \\
\hline 71 & 35.22 & 1425 & 1436 & Ethyl octanoate ${ }^{4}$ & 3.97 & (4) & 2.61 & (4) & 4.11 & (7) & 4.39 & (14) & 72.42 & (12) & 42.25 & (4) & 16.89 & (16) & 16.83 & (17) & 47.25 & (4) & 53.46 & (11) \\
\hline 78 & 37.64 & 1465 & 1441 & Isopentyl hexanoate & - & & - & & & & & & & & 18.74 & (2) & - & & & & & & 6.28 & (9) \\
\hline 79 & 37.81 & 1468 & 148 & Ethyl 3-hydroxybutanoate ${ }^{4}$ & - & & - & & 181.49 & $(8)$ & 67.28 & (15) & 220.28 & (7) & 154.85 & (5) & 46.42 & (9) & 20.54 & (16) & 7.41 & (15) & 1.67 & (16) \\
\hline 89 & 41.82 & 1532 & 1533 & Hexyl butanoate & - & & - & & 12.44 & (14) & 7.26 & (4) & - & & - & & - & & & & & & - & \\
\hline 100 & 45.96 & 1597 & 1591 & Methyl decanoate & - & & 0.38 & (4) & 6.96 & (10) & 25.22 & (5) & - & & - & & 8.15 & (18) & 5.16 & (10) & 17.67 & (8) & 1.84 & (15) \\
\hline 104 & 47.59 & 1624 & 1610 & Butyl octanoate & - & & 0.54 & (5) & & & 7.06 & (18) & - & & - & & - & & 0.98 & (7) & & & 9.51 & (14) \\
\hline 108 & 48.69 & 1643 & 1636 & Ethyl decanoate ${ }^{4}$ & - & & - & & 102.43 & $3(12)$ & 84.04 & (8) & - & & - & & 60.17 & (19) & 6.74 & (15) & 6.79 & (12) & 19.02 & (13) \\
\hline 110 & 49.08 & 1650 & 1648 & Ethyl benzoate ${ }^{4}$ & 2.14 & (3) & 1.99 & (2) & - & & 1.68 & (2) & & & - & & - & & 2.04 & (1) & 2.39 & (7) & 0.97 & (1) \\
\hline 112 & 49.54 & 1657 & 1664 & Ethyl 3-hydroxyhexanoate ${ }^{4}$ & & & - & & 5.45 & (17) & 40.42 & (5) & 6.28 & (4) & 27.82 & (12) & 1.93 & (7) & 58.21 & (9) & 5.41 & (16) & 7.43 & (9) \\
\hline
\end{tabular}


Table 1. Cont

\begin{tabular}{|c|c|c|c|c|c|c|c|c|c|c|c|c|c|c|c|c|c|c|c|c|c|c|c|c|}
\hline \multirow{4}{*}{$\begin{array}{c}\begin{array}{c}\text { Peak } \\
\mathbf{n}^{\circ}\end{array} \\
116\end{array}$} & \multirow{4}{*}{$\begin{array}{c}\begin{array}{c}\text { RT } \\
(\mathbf{m i n})^{1}\end{array} \\
51.36\end{array}$} & \multirow{4}{*}{$\begin{array}{c}\mathrm{KI}_{\mathrm{Cal}}{ }^{2} \\
1687\end{array}$} & \multirow{4}{*}{$\begin{array}{c}\mathbf{K I}_{\mathrm{Lit}}{ }^{3} \\
1683\end{array}$} & \multirow{4}{*}{$\begin{array}{l}\text { Chemical Groups } \\
\text { Benzyl butanoate }\end{array}$} & \multicolumn{20}{|c|}{ GC Peak Area $\left(\times 10^{6}\right)($ RSD) } \\
\hline & & & & & \multicolumn{4}{|c|}{ Grape } & \multicolumn{4}{|c|}{ Red Fruits } & \multicolumn{4}{|c|}{ Orange } & \multicolumn{4}{|c|}{ Pear } & \multicolumn{4}{|c|}{ Apple } \\
\hline & & & & & \multicolumn{2}{|c|}{ Fresh } & \multicolumn{2}{|c|}{ Processed } & \multicolumn{2}{|c|}{ Fresh } & \multicolumn{2}{|c|}{ Processed } & Fre & & Proce & ssed & Fres & & Proces & ssed & Fres & & Proce & ssed \\
\hline & & & & & 18.43 & (7) & 2.27 & (9) & 2.01 & (14) & - & & - & & - & & - & & - & & 17.49 & (8) & 2.58 & (13) \\
\hline 117 & 52.22 & 1700 & 1689 & Diethyl succinate ${ }^{4}$ & - & & 1.04 & (14) & 3.19 & (14) & - & & - & & - & & 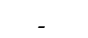 & & - & & 1.48 & (11) & - & \\
\hline 120 & 52.69 & 1708 & 1694 & Ethyl 9-decenoate & 6.83 & (7) & 1.03 & (4) & 66.22 & (18) & 204.61 & (5) & 184.42 & (9) & 93.89 & (8) & 4.33 & (3) & 10.33 & (7) & 190.78 & (7) & 74.42 & (9) \\
\hline 130 & 55.09 & 1747 & 1745 & Methyl salicylate ${ }^{4}$ & 53.55 & (4) & 27.22 & (5) & 3.86 & (12) & 1.97 & (3) & - & & 4.59 & (10) & - & & 1.59 & (5) & - & & 0.78 & (9) \\
\hline 134 & 56.42 & 1768 & 1729 & 3-Hexenyl hexanoate & & & & & 0.68 & (19) & 2.06 & (5) & 6.97 & (2) & 3.53 & (10) & 20.76 & (16) & 1.23 & (2) & 3.25 & (8) & 4.63 & (14) \\
\hline 137 & 57.41 & 1783 & 1803 & 2-Phenylethyl acetate 4 & 27.70 & (7) & 2.12 & (11) & 59.28 & (13) & 16.31 & (7) & 12.34 & (17) & 7.78 & (8) & 4.50 & (8) & 12.12 & (2) & 1.06 & (2) & 10.47 & (8) \\
\hline 141 & 58.86 & 1807 & 1821 & Benzyl propanoate & - & & - & & 0.54 & (7) & 1.98 & (4) & - & & - & & 168.13 & (14) & 42.07 & (5) & $10.62^{\mathrm{b}}$ & (18) & $10.74^{\mathrm{b}}$ & (4) \\
\hline 146 & 60.32 & 1839 & 1837 & Ethyl dodecanoate 4 & - & & 1.07 & (9) & - & & 22.39 & (18) & - & & - & & - & & 5.47 & (6) & - & & 27.04 & (12) \\
\hline 148 & 61.85 & 1872 & 1872 & Geranyl butanoate & 4.23 & (4) & 2.10 & (18) & 0.74 & (17) & 9.42 & (15) & - & & - & & 9.88 & (4) & 2.37 & (11) & - & & 22.58 & (5) \\
\hline 151 & 63.49 & 1906 & 1895 & Geranyl isovalerate & - & & 1.71 & (13) & 0.50 & (6) & - & & - & & - & & 64.89 & (14) & 2.89 & (4) & - & & - & \\
\hline 154 & 65.54 & 1944 & 1960 & Geranyl valerate & - & & & & & & & & - & & - & & 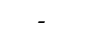 & & - & & 1.98 & (17) & - & \\
\hline 155 & 66.22 & 1956 & 1974 & Methyl jasmonate & 11.10 & (4) & 21.96 & (11) & 15.26 & (7) & 29.99 & (9) & - & & - & & - & & - & & - & & - & \\
\hline 161 & 73.02 & 2126 & 2114 & Bornyl benzoate & - & & - & & (2) & & - & & 7.79 & (11) & - & & 4.47 & (14) & 18.77 & (10) & - & & - & \\
\hline 163 & 74.72 & 2135 & 2139 & Ethyl cinnamate 4 & - & & 2.89 & (9) & 2.15 & (4) & 6.64 & (7) & $1.02^{\mathrm{c}}$ & (14) & $0.98^{c}$ & (2) & 37.80 & (19) & 1.45 & (10) & 3.40 & (17) & 2.97 & (7) \\
\hline 168 & 77.99 & 2154 & 2171 & Diethyl 2-hydroxyglutarate & 1.28 & (2) & 0.52 & (12) & 0.39 & (5) & 0.15 & (4) & - & & - & & 4.77 & (10) & 2.15 & (16) & 2.34 & (8) & 2.03 & (10) \\
\hline & & & & $\begin{array}{c}\% \text { RPA } \\
\text { Alcohols }\end{array}$ & 25.98 & & 18.45 & & 64.16 & & 50.29 & & 23.58 & & 20.90 & & 58.84 & & 45.15 & & 55.00 & & 57.83 & \\
\hline 6 & 7.62 & 952 & 929 & Ethanol ${ }^{4}$ & 106.00 & (4) & 82.28 & (9) & 51.19 & (15) & 119.72 & (9) & 67.62 & (12) & 89.99 & (8) & 185.09 & (14) & 111.82 & (12) & 93.32 & (6) & 136.76 & (14) \\
\hline 14 & 11.29 & 1023 & 1037 & 1-Propanol 4 & 40.25 & (3) & 11.05 & (5) & 52.66 & (7) & 84.80 & (4) & 4.26 & (3) & 3.98 & (2) & 4.17 & (7) & 3.63 & (13) & 10.21 & (3) & 24.98 & (2) \\
\hline 27 & 16.86 & 1142 & 1099 & 2-Butanol ${ }^{4}$ & 2.80 & (5) & 0.35 & (6) & - & & - & & 18.87 & (6) & 21.16 & (6) & 11.99 & (17) & 6.14 & (5) & 24.91 & (4) & 1.80 & (9) \\
\hline 37 & 20.56 & 1201 & 1206 & 3-Methyl-1-butanol 4 & 1.83 & (2) & 0.99 & (7) & 2.15 & (3) & 4.25 & (2) & 40.21 & (2) & 55.17 & (4) & 8.39 & (18) & 7.23 & (2) & 109.54 & (4) & 99.08 & (2) \\
\hline 59 & 29.87 & 1344 & 1332 & 2 -Heptanol ${ }^{4}$ & 33.40 & (6) & 30.15 & (8) & 13.20 & (10) & 53.42 & (4) & 36.18 & (17) & 4.01 & (1) & 160.92 & (10) & 296.92 & (8) & 6.78 & (19) & 13.78 & (3) \\
\hline 60 & 30.59 & 1355 & 1360 & $1-\mathrm{Hexanol}^{4}$ & - & & & & & & 6.89 & (5) & - & & - & & - & & - & & - & & 7.00 & (10) \\
\hline 62 & 31.93 & 1374 & 1379 & 3-Ethoxypropanol & 2.55 & (6) & 0.83 & (10) & 7.36 & $(10)$ & 18.74 & (9) & 6.42 & (17) & 8.26 & (2) & - & & - & & - & & 19.92 & (2) \\
\hline 67 & 33.31 & 1394 & 1391 & 3-Hexen-1-ol isomer ${ }^{4}$ & 6.95 & (5) & 0.89 & (3) & 23.25 & (8) & 66.75 & (5) & 13.55 & (13) & 25.46 & (5) & 3.37 & (16) & 4.28 & (8) & 17.11 & (10) & 28.55 & (2) \\
\hline 74 & 36.18 & 1441 & 1445 & 1-Octen-3-ol ${ }^{4}$ & 3.04 & (14) & 2.20 & (3) & 4.27 & (2) & 3.33 & (4) & 8.90 & (7) & 6.30 & (15) & 8.57 & (5) & 21.73 & (6) & 1.19 & (18) & 15.40 & (3) \\
\hline 75 & 36.37 & 1444 & 1467 & Heptanol $^{4}$ & - & & - & & 27.03 & (3) & 9.14 & (8) & 12.33 & (18) & 9.70 & (3) & - & & - & & 8.51 & (6) & 13.99 & (15) \\
\hline 81 & 38.61 & 1480 & 1487 & 2-Ethyl-1-hexanol ${ }^{4}$ & 10.47 & (9) & 9.44 & (13) & 1.56 & (9) & 7.55 & (9) & 2.82 & (10) & 10.25 & (2) & 4.43 & (13) & 30.86 & (5) & 5.58 & (8) & 22.00 & (7) \\
\hline 91 & 42.69 & 1546 & 1553 & $1_{1-O c t a n o l}{ }^{4}$ & 6.62 & (2) & 7.29 & (8) & 20.37 & (4) & 76.80 & (4) & 72.34 & (9) & 22.57 & (3) & 27.20 & (5) & 68.14 & (4) & 24.58 & (11) & 75.42 & (2) \\
\hline 109 & 48.80 & 1645 & 1624 & 1-Nonanol & 3.45 & (12) & 3.78 & (18) & 4.67 & $(20)$ & 29.29 & (6) & 23.40 & (7) & 46.54 & (9) & & & - & & - & & - & \\
\hline 147 & 60.80 & 1850 & 1865 & Benzyl alcohol ${ }^{4}$ & 1.96 & (14) & 3.47 & (12) & 1.51 & (13) & 30.96 & (3) & - & & - & & 9.62 & (15) & 5.55 & (2) & - & & - & \\
\hline 150 & 62.97 & 1896 & 1925 & Phenylethyl alcohol ${ }^{4}$ & 19.48 & (6) & 22.53 & (7) & 1.92 & (5) & 6.72 & (10) & 3.10 & (8) & 8.23 & (4) & 13.47 & (3) & 31.77 & (17) & 11.77 & (4) & 10.93 & (13) \\
\hline 156 & 67.46 & 1978 & 1952 & 1-Tridecanol & - & & 0.77 & (4) & - & & - & & - & & - & & - & & - & & - & & 7.71 & (17) \\
\hline & & & & \% RPA & 18.14 & & 19.97 & & 7.14 & & 15.08 & & 10.05 & & 9.67 & & 16.21 & & 20.74 & & 10.14 & & 15.60 & \\
\hline 2 & 6.46 & 921 & - & $\begin{array}{l}\text { Carbonyl compounds } \\
\text { Butanal }^{4}\end{array}$ & - & & - & & 5.47 & (18) & 9.43 & (17) & 4.25 & (2) & 9.50 & (3) & 3.19 & (5) & 7.42 & (16) & 1.70 & (15) & 5.24 & (11) \\
\hline 4 & 7.19 & 941 & 926 & 2-Methylbutanal & 3.34 & (2) & 1.75 & (1) & 2.34 & (7) & 1.97 & (2) & - & & - & & - & & - & & 0.26 & (11) & 2.38 & (9) \\
\hline 5 & 7.33 & 944 & 936 & 3-Methylbutanal ${ }^{4}$ & 4.63 & (4) & 1.69 & (14) & & & & & - & & - & & & & - & & & & & \\
\hline 9 & 8.98 & 982 & 973 & 2-Pentanone & - & & - & & $\frac{1.08}{d}$ & (2) & $\frac{0.96}{d}$ & (3) & 7.34 & (5) & 2.14 & (12) & 30.36 & (5) & 2.87 & (8) & 6.22 & (5) & 0.77 & (4) \\
\hline 20 & 13.31 & 1072 & 1084 & & 17.26 & (11) & 2.06 & (18) & 18.35 & (7) & 9.78 & (12) & 18.53 & (12) & 14.47 & (7) & - & & - & & 18.57 & (18) & 7.93 & (6) \\
\hline 24 & 15.93 & 1125 & 1131 & 2-Pentenal & - & & 2.44 & (9) & - & & 3.02 & (13) & - & & - & & - & & - & & - & & 1.28 & (15) \\
\hline 32 & 18.95 & 1177 & 1170 & 2-Heptanone & - & & 2.12 & (1) & & & 1.27 & (18) & - & & - & & - & & 1.08 & (10) & - & & 3.51 & (16) \\
\hline 33 & 19.13 & 1179 & 1174 & Heptanal ${ }^{4}$ & 21.96 & (10) & 24.03 & (7) & 5.36 & (17) & 12.33 & (5) & - & & - & & - & & 0.34 & (8) & 1.66 & (13) & 4.05 & (7) \\
\hline
\end{tabular}


Table 1. Cont

\begin{tabular}{|c|c|c|c|c|c|c|c|c|c|c|c|c|c|c|c|c|c|c|c|c|c|c|c|c|}
\hline \multirow{4}{*}{$\begin{array}{c}\begin{array}{c}\text { Peak } \\
\mathbf{n}^{\circ}\end{array} \\
36\end{array}$} & \multirow{4}{*}{$\begin{array}{c}\begin{array}{c}\mathrm{RT} \\
(\mathrm{min})^{1}\end{array} \\
20.22\end{array}$} & \multirow{4}{*}{$\begin{array}{c}\mathrm{KI}_{\mathrm{Cal}}{ }^{2} \\
1196\end{array}$} & \multirow{4}{*}{$\begin{array}{r}\mathrm{KI}_{\mathrm{Lit}}{ }^{3} \\
1192\end{array}$} & \multirow{4}{*}{$\begin{array}{l}\text { Chemical Groups } \\
\text { 2-Hexenal isomer }\end{array}$} & \multicolumn{20}{|c|}{ GC Peak Area $\left(\times 10^{6}\right)($ RSD) } \\
\hline & & & & & \multicolumn{4}{|c|}{ Grape } & \multicolumn{4}{|c|}{ Red Fruits } & \multicolumn{4}{|c|}{ Orange } & \multicolumn{4}{|c|}{ Pear } & \multicolumn{4}{|c|}{ Apple } \\
\hline & & & & & \multicolumn{2}{|c|}{ Fresh } & \multicolumn{2}{|c|}{ Processed } & Fres & & Proces & ssed & Fre & & Proces & sed & Fre & & Proce & ssed & Fre & & Proce & ssed \\
\hline & & & & & 22.33 & (1) & 25.43 & (6) & 36.36 & (5) & 27.90 & (1) & 10.24 & (3) & 20.25 & (2) & 3.27 & (11) & 38.82 & (4) & 8.61 & (16) & 20.50 & (13) \\
\hline 38 & 21.17 & 1211 & 1200 & 2-Hexenal isomer & 4.66 & (10) & 2.89 & (10) & 17.01 & (15) & 19.74 & (5) & & & - & & 21.20 & (5) & 3.08 & (2) & 31.28 & (13) & & \\
\hline 43 & 23.49 & 1248 & 1244 & 3-Octanone 4 & 14.11 & (9) & 1.51 & (9) & 0.52 & (12) & 5.30 & (8) & - & & - & & 0.41 & (1) & - & & - & & 1.90 & (12) \\
\hline 46 & 25.58 & 1279 & 1272 & 3-Hydroxy-2-butanone & 9.30 & (9) & 0.97 & (4) & - & & - & & - & & - & & - & & 13.09 & (10) & - & & 1.18 & (17) \\
\hline 47 & 25.73 & 1281 & 1280 & Octanal $^{4}$ & - & & 7.10 & (6) & 8.63 & (3) & 3.73 & (10) & 99.19 & (13) & 122.34 & (4) & - & & - & & - & & 18.05 & (5) \\
\hline 48 & 26.58 & 1292 & 1287 & 1-Octen-3-one 4 & - & & 18.29 & (7) & - & & - & & - & & - & & - & & - & & - & & 4.89 & (9) \\
\hline 53 & 27.92 & 1313 & 1299 & 2-Heptenal isomer & - & & - & & 6.75 & (3) & 4.23 & (6) & 4.53 & (16) & 10.89 & (9) & 1.57 & (15) & 4.26 & (3) & 6.51 & (4) & 1.02 & (6) \\
\hline 55 & 28.90 & 1329 & 1319 & 6-Methyl-5-hepten-2-one & 25.18 & (7) & 3.10 & (15) & - & & - & & 3.57 & (3) & 8.24 & (11) & 24.52 & (10) & 12.91 & (6) & 119.93 & (11) & 40.24 & (8) \\
\hline 63 & 32.20 & 1378 & 1388 & 2-Nonanone & 2.24 & (1) & 16.47 & (8) & - & & - & & 4.74 & (1) & 1.91 & (7) & 0.98 & (2) & 1.63 & (9) & 2.22 & (10) & 2.83 & (1) \\
\hline 65 & 32.51 & 1383 & 1385 & Nonanal $^{4}$ & 19.70 & (15) & 0.91 & (13) & 72.47 & (9) & 18.60 & (13) & 46.59 & (7) & 41.59 & (13) & 15.56 & (1) & 14.10 & (3) & 10.13 & (13) & 17.48 & (10) \\
\hline 66 & 33.17 & 1392 & 1401 & 2,4-Heptadienal isomer & & & & & 10.93 & (1) & 7.66 & (4) & 2.25 & (1) & 2.86 & (6) & - & & - & & 1.20 & (4) & 1.94 & (5) \\
\hline 70 & 34.73 & 1417 & 1408 & 2-Octenal isomer & 7.59 & (15) & 0.43 & (5) & 1.32 & (14) & 0.96 & (6) & 3.58 & (19) & 5.86 & (2) & 18.06 & (7) & - & & 35.95 & (8) & 14.73 & (9) \\
\hline 82 & 38.78 & 1483 & 1458 & Methional $^{4}$ & 1.41 & (3) & 2.42 & (8) & 3.53 & (5) & 6.54 & (14) & 10.12 & (14) & 15.97 & (3) & 1.13 & (18) & 13.33 & (7) & 0.67 & (7) & 2.14 & (2) \\
\hline 83 & 39.12 & 1488 & 1484 & Decanal $^{4}$ & 40.95 & (6) & 14.28 & (15) & 37.57 & (13) & 16.69 & (12) & 84.32 & (7) & 35.24 & (1) & 15.61 & (3) & 11.92 & (15) & 14.23 & (18) & 28.73 & (8) \\
\hline 86 & 40.58 & 1512 & 1495 & Benzaldehyde 4 & 25.94 & (12) & 21.09 & (8) & 5.38 & (17) & 18.16 & (2) & 2.67 & (18) & 14.01 & (12) & 16.05 & (8) & 3.19 & (3) & 6.91 & (11) & 24.45 & (2) \\
\hline 87 & 41.30 & 1524 & 1527 & 2-Nonenal isomer 4 & - & & - & & 6.10 & (9) & 9.37 & (14) & 10.19 & (11) & 16.22 & (2) & 5.74 & (13) & 1.59 & (8) & 3.01 & (12) & 9.74 & (19) \\
\hline 95 & 44.40 & 1573 & 1575 & 2,6-Nonadienal isomer & 1.46 & (1) & 6.51 & (5) & $\begin{array}{c}1.08 \\
\mathrm{e}\end{array}$ & (15) & $\begin{array}{c}0.98 \\
\mathrm{e}\end{array}$ & (1) & - & & - & & - & & 2.07 & (16) & - & & - & \\
\hline 96 & 44.77 & 1579 & 1561 & 6-Methyl-3,5-heptadiene-2-one & - & & 2.47 & (12) & 12.57 & (19) & 6.44 & (2) & - & & - & & - & & 3.18 & (15) & - & & 4.35 & (9) \\
\hline 106 & 48.08 & 1633 & 1645 & Acetophenone 4 & 9.26 & (1) & 1.05 & (17) & 9.61 & (5) & 7.82 & (9) & - & & - & & 17.96 & (18) & 3.14 & (2) & - & & - & \\
\hline 127 & 54.61 & 1739 & 1710 & 2,4-Decadienal ${ }^{4}$ & - & & 3.17 & (14) & 1.66 & (11) & 6.89 & (17) & - & & - & & 10.06 & (6) & 9.62 & (15) & - & & 34.88 & (9) \\
\hline 129 & 54.96 & 1745 & 1753 & Ethyl benzaldehyde 4 & - & & - & & - & & - & & - & & 10.48 & (4) & - & & 2.75 & (9) & - & & - & \\
\hline 136 & 57.07 & 1778 & 1758 & 2,4-Decadienal & - & & 0.23 & (5) & - & & - & & 11.22 & (6) & 7.15 & (2) & 5.39 & (11) & 3.27 & (3) & 8.91 & (15) & 2.37 & (4) \\
\hline 164 & 75.14 & 2138 & 2103 & $\gamma$-Decalactone ${ }^{4}$ & - & & 1.07 & (5) & - & & 10.56 & (9) & & & - & & - & & 1.36 & (11) & - & & - & \\
\hline 166 & 76.38 & 2145 & 2112 & Hexadecanone & - & & 0.61 & (12) & 0.60 & (10) & 2.75 & (11) & - & & - & & - & & - & & - & & - & \\
\hline 169 & 80.45 & 2167 & 2153 & Palmitaldehyde & - & & - & & 25.00 & (12) & 17.37 & (14) & - & & - & & - & & - & & - & & - & \\
\hline & & & & $\begin{array}{c}\% \text { RPA } \\
\text { Terpenoids }\end{array}$ & 17.57 & & 18.62 & & 9.80 & & 6.70 & & 10.48 & & 10.53 & & 7.08 & & 5.47 & & 8.99 & & 8.39 & \\
\hline 12 & 10.45 & 1000 & 1032 & $\begin{array}{l}\text { Terpenoids } \\
\alpha \text {-Pinene }{ }^{4}\end{array}$ & - & & - & & - & & - & & 79.43 & (4) & 38.60 & (5) & - & & 5.59 & (16) & 655 & (18) & 147 & (6) \\
\hline 17 & 12.26 & 1047 & 1075 & Camphene & - & & - & & - & & - & & 1.17 & (13) & 0.98 & (6) & - & & 1.03 & (3) & - & & & \\
\hline 21 & 14.03 & 108 & 111 & $\beta$-Pinene ${ }^{4}$ & - & & - & & - & & - & & 4.84 & (3) & - & & - & & 6.22 & (14) & - & & 5.23 & (11) \\
\hline 23 & 15.90 & 1124 & 1148 & $\delta$-3-Carene & - & & - & & - & & - & & 9.57 & (11) & 8.96 & (5) & - & & - & & - & & - & \\
\hline 29 & 17.73 & 1157 & 1141 & $\beta$-Myrcene ${ }^{4}$ & - & & - & & 0.72 & (7) & 7.17 & (5) & 16.13 & (12) & 4.96 & (2) & - & & - & & - & & - & \\
\hline 35 & 19.68 & 1188 & 1198 & Limonene ${ }^{4}$ & 5.61 & (5) & 1.16 & (6) & 0.96 & (4) & 7.72 & (10) & 359.17 & (9) & 203.95 & (3) & 14.12 & (3) & 1.81 & (6) & 9.31 & (4) & 11.47 & (6) \\
\hline 39 & 21.92 & 1224 & 1225 & Ocimene & - & & - & & - & & & & 55.58 & (3) & 99.12 & (3) & 8.89 & (14) & 12.83 & (7) & - & & 12.73 & (12) \\
\hline 42 & 22.85 & 1239 & 123 & $\gamma$-Terpinene & 20.74 & (7) & 1.75 & (8) & - & & - & & 20.58 & (16) & 8.16 & (4) & - & & - & & - & & - & \\
\hline 45 & 25.06 & 1271 & 1261 & $p$-Cymene ${ }^{4}$ & - & & - & & - & & - & & 76.29 & (9) & 99.40 & (11) & - & & - & & - & & - & \\
\hline 58 & 29.73 & 1342 & 1337 & Rose oxide isomer & - & & - & & 1.05 & (5) & 1.36 & (12) & & & - & & - & & 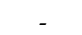 & & & & . & \\
\hline 72 & 35.47 & 142 & 1423 & Linalool oxide isomer & - & & - & & 2.98 & (2) & 3.17 & (15) & 15.28 & (7) & 12.36 & (3) & 2.14 & (5) & 24.11 & (11) & 10.13 & (2) & 5.48 & (1) \\
\hline 73 & 35.58 & 1431 & 1449 & Dihydrolinalool & 83.01 & (5) & 48.23 & $(8)$ & - & & - & & - & & - & & - & & - & & - & & - & \\
\hline 80 & 37.90 & 1469 & 1488 & Citronellal $^{4}$ & 6.33 & (2) & 4.23 & (5) & - & & & & 26.50 & (15) & 12.15 & (6) & - & & & & 0.95 & (17) & 1.20 & (8) \\
\hline 84 & 39.77 & 1498 & 1491 & Camphor & 17.77 & (15) & 2.93 & (10) & 3.26 & (10) & 3.21 & (11) & 7.98 & (5) & 8.22 & (4) & 8.05 & (14) & 25.32 & (6) & 1.79 & (7) & 7.35 & (11) \\
\hline 85 & 40.32 & 1507 & 1506 & Isoborneol & 7.60 & (9) & 6.11 & (2) & - & & & & - & & - & & 24.88 & (15) & 7.13 & (2) & 26.74 & (7) & 11.44 & (8) \\
\hline 88 & 41.61 & 1529 & 1516 & Isocitral & 16.34 & (15) & 1.63 & (6) & 10.86 & (15) & 5.51 & (3) & - & & - & & 3.52 & (3) & 5.51 & (10) & 0.96 & (13) & 3.48 & (13) \\
\hline 90 & 42.18 & 1538 & 1537 & Linalool $^{4}$ & 60.66 & (2) & 18.25 & (4) & 254.57 & (17) & 375.08 & (4) & 46.40 & (17) & 198.03 & (4) & 5.54 & (9) & 24.57 & (8) & 7.48 & (4) & 41.07 & (6) \\
\hline 92 & 43.37 & 1557 & 1550 & Dihydrocarvone & - & & - & & - & & - & & 40.21 & (3) & 58.42 & (5) & 2.76 & (2) & 4.43 & (9) & 1.32 & (13) & 3.73 & (11) \\
\hline 94 & 44.12 & 1569 & 1574 & Fenchyl alcohol & - & & - & & 4.67 & (12) & 4.88 & (10) & 64.49 & (6) & 24.65 & (6) & 20.45 & (18) & 3.72 & (11) & 15.76 & (4) & 30.35 & (5) \\
\hline
\end{tabular}


Table 1. Cont

\begin{tabular}{|c|c|c|c|c|c|c|c|c|c|c|c|c|c|c|c|c|c|c|c|c|c|c|c|c|}
\hline \multirow{4}{*}{$\begin{array}{c}\begin{array}{c}\text { Peak } \\
\mathbf{n}^{\circ}\end{array} \\
97\end{array}$} & \multirow{4}{*}{$\begin{array}{c}\begin{array}{c}\text { RT } \\
(\mathbf{m i n})^{1}\end{array} \\
45.21\end{array}$} & \multirow{4}{*}{$\begin{array}{r}\mathrm{KI}_{\mathrm{Cal}}{ }^{2} \\
1585\end{array}$} & \multirow{4}{*}{$\begin{array}{r}\mathbf{K I}_{\mathbf{L i t}}{ }^{3} \\
1580\end{array}$} & \multirow{4}{*}{$\begin{array}{c}\text { Chemical Groups } \\
\text { Bornyl acetate }\end{array}$} & \multicolumn{20}{|c|}{ GC Peak Area $\left(\times 10^{6}\right)($ RSD) } \\
\hline & & & & & \multicolumn{4}{|c|}{ Grape } & \multicolumn{4}{|c|}{ Red Fruits } & \multicolumn{4}{|c|}{ Orange } & \multicolumn{4}{|c|}{ Pear } & \multicolumn{4}{|c|}{ Apple } \\
\hline & & & & & \multicolumn{2}{|c|}{ Fresh } & \multicolumn{2}{|c|}{ Processed } & \multicolumn{2}{|c|}{ Fresh } & Proce & ssed & Fres & & Proce & ssed & Fre & & Proce & sed & Fres & & Proce & ssed \\
\hline & & & & & - & & - & & 3.18 & (13) & 37.19 & (1) & 12.33 & (12) & 16.01 & (6) & 10.25 & (3) & 14.70 & (3) & 1.95 & (15) & 7.62 & (10) \\
\hline 98 & 45.58 & 1591 & 1562 & Isodihydrocarveol & - & & - & & - & & - & & 25.67 & (6) & 65.65 & (5) & 0.78 & (4) & 1.84 & (7) & 1.64 & (13) & 9.09 & (5) \\
\hline 99 & 45.89 & 1596 & 1596 & Mrytenal & 39.62 & (6) & 6.52 & (3) & - & & & & 12.08 & (6) & 11.93 & (14) & - & & - & & & & & \\
\hline 101 & 46.15 & 1599 & 1598 & $\beta$-Ciclocitral ${ }^{4}$ & 13.87 & (7) & 1.25 & (2) & 24.00 & (12) & 6.40 & (4) & 1.26 & (9) & 16.60 & (8) & 32.96 & (4) & 11.92 & (7) & 62.18 & (11) & 9.01 & (7) \\
\hline 102 & 46.53 & 1606 & 1602 & Citral $^{4}$ & 11.21 & (14) & 5.50 & (6) & 2.98 & (2) & 3.17 & (5) & 5.01 & (13) & 7.72 & (6) & 4.96 & (3) & 5.21 & (3) & 1.53 & (1) & 2.96 & (2) \\
\hline 103 & 46.99 & 1614 & 1646 & $\beta$-Terpineol ${ }^{4}$ & 53.04 & (11) & 20.97 & (4) & 34.86 & (15) & 26.29 & (7) & 6.56 & (8) & 14.37 & (10) & 10.01 & (3) & 2.70 & (8) & 1.21 & (2) & 4.88 & (7) \\
\hline 105 & 47.70 & 1626 & 1648 & Safranal & - & & - & & - & & - & & 49.88 & (8) & 55.30 & (12) & - & & - & & - & & - & \\
\hline 107 & 48.44 & 1639 & 1639 & Carvone & - & & - & & - & & & & - & & - & & 4.21 & (2) & 4.02 & (5) & 9.15 & (8) & 2.16 & (18) \\
\hline 111 & 49.37 & 1654 & 1655 & Estragole & - & & - & & 8.27 & (15) & 4.27 & (10) & - & & - & & 41.13 & (16) & 32.61 & (9) & 31.53 & (14) & 13.11 & (9) \\
\hline 113 & 50.21 & 1668 & 1648 & $\beta$-Farnesene isomer & - & & - & & 14.23 & (5) & 8.96 & (3) & 54.22 & (2) & 90.62 & (8) & 5.01 & (3) & 16.44 & (12) & 1.57 & (17) & 2.33 & (12) \\
\hline 114 & 50.80 & 1678 & 1688 & $\alpha$-Terpineol ${ }^{4}$ & 21.09 & (6) & 13.47 & (9) & 9.70 & (1) & 12.44 & (9) & 40.86 & (7) & 29.87 & (7) & 15.12 & (4) & 11.91 & (11) & 10.18 & (7) & 15.52 & (1) \\
\hline 115 & 51.03 & 1681 & 1667 & Neral & - & & - & & & & & & & & - & & 4.06 & (8) & 2.06 & (3) & 5.59 & (5) & 1.34 & (8) \\
\hline 118 & 52.30 & 1701 & 1705 & Germacrene D & - & & - & & 2.76 & (9) & 1.96 & (5) & 128.16 & (4) & 18.84 & (5) & 1.77 & (17) & 1.66 & (12) & 9.10 & (8) & 11.36 & (4) \\
\hline 119 & 52.57 & 1706 & 1715 & Geranial isomer & - & & - & & - & & - & & - & & - & & 34.53 & (11) & 19.82 & (5) & - & & - & \\
\hline 121 & 53.05 & 1714 & 1718 & Naphthalene & 4.28 & (1) & 8.10 & (13) & - & & - & & - & & - & & 3.00 & (6) & 3.80 & (14) & 17.96 & (2) & 19.54 & (5) \\
\hline 122 & 53.26 & 1717 & 1716 & TDN & 12.46 & (6) & 1.79 & (4) & - & & - & & 3.16 & (11) & - & & - & & - & & & & 0.99 & (9) \\
\hline 123 & 53.65 & 1724 & 1726 & Valencene & - & & - & & - & & - & & 24.14 & (7) & - & & 11.69 & (7) & - & & 9.51 & (10) & - & \\
\hline 124 & 53.91 & 1728 & 1725 & $\alpha$-Farnesene & - & & - & & 13.75 & (19) & 20.96 & (5) & 27.25 & (4) & 21.86 & (7) & 18.58 & (10) & 27.96 & (12) & 419.32 & (3) & 8.16 & (5) \\
\hline 125 & 54.10 & 1731 & 1714 & $\alpha$-Muurolene & - & & - & & - & & - & & 38.90 & (1) & 31.01 & (8) & 12.77 & (9) & 1.80 & (1) & - & & - & \\
\hline 126 & 54.27 & 1734 & 1711 & Geranyl acetate & - & & - & & - & & - & & 31.54 & (4) & 66.51 & (10) & - & & & & 23.65 & (12) & 13.37 & (13) \\
\hline 128 & 54.74 & 1741 & 1715 & Geranial isomer & 14.43 & (13) & 0.50 & (3) & - & & - & & 50.82 & (13) & 72.06 & (10) & 0.98 & (2) & 1.97 & (2) & 3.61 & (5) & 5.64 & (12) \\
\hline 131 & 55.43 & 1752 & 1751 & Carvone & - & & - & & - & & - & & 66.83 & (3) & 103.53 & (8) & - & & - & & - & & - & \\
\hline 132 & 55.82 & 1758 & 1759 & Cumin aldehyde & - & & - & & 3.88 & (4) & 9.76 & (7) & 2.48 & (16) & 15.89 & (12) & - & & - & & - & & - & \\
\hline 133 & 55.95 & 1760 & 1762 & $\beta$-Citronellol ${ }^{4}$ & - & & - & & 10.91 & (16) & 8.67 & (10) & & & - & & 7.43 & (5) & 34.98 & (5) & 23.08 & (15) & 1.84 & (11) \\
\hline 135 & 56.53 & 1769 & 1770 & Nerol & 7.34 & $(4)$ & 4.67 & (7) & - & & - & & 82.71 & (11) & 87.95 & (9) & - & & - & & 2.87 & (1) & 1.59 & (3) \\
\hline 138 & 57.50 & 1784 & 1765 & Perillaldehyde & 17.32 & (10) & 10.26 & (9) & 8.62 & (4) & 12.92 & (5) & 1.36 & (3) & 2.15 & (4) & 1.83 & (9) & 45.48 & (10) & 19.86 & (6) & 22.03 & (10) \\
\hline 139 & 58.01 & 1792 & 1813 & $\beta$-Damascenone ${ }^{4}$ & 6.98 & (3) & 10.03 & (13) & 2.41 & (14) & 20.16 & (2) & 1.34 & (9) & 1.21 & (2) & 5.65 & (11) & 4.37 & (4) & 1.10 & (8) & 0.92 & (5) \\
\hline 140 & 58.38 & 1798 & 1786 & Cadinadiene & - & & - & & 1.74 & (15) & 2.76 & (1) & 16.07 & (18) & 33.65 & (12) & 22.32 & (17) & 17.16 & (6) & - & & - & \\
\hline 142 & 59.08 & 1812 & 1809 & $\alpha$-Ionone ${ }^{4}$ & - & & 0.53 & (6) & - & & 8.66 & (6) & & & & & & & & & & & 10.22 & (9) \\
\hline 144 & 59.60 & 1824 & 1840 & Geranyl acetone isomer & 1.76 & (1) & 3.27 & (4) & - & & . & & 109.91 & (15) & 73.38 & (7) & 7.53 & (1) & 6.58 & (6) & - & & 27.62 & (15) \\
\hline 145 & 60.10 & 1834 & 1839 & trans-carveol & - & & 2.29 & (7) & - & & - & & 8.23 & (16) & 11.22 & (9) & 9.89 & (7) & 4.08 & (9) & - & & - & \\
\hline 149 & 62.13 & 1878 & 1847 & Geraniol $^{4}$ & - & & - & & 9.17 & (1) & 11.90 & (10) & & & - & & 16.53 & (8) & 6.74 & (14) & - & & - & \\
\hline 152 & 64.72 & 1929 & 1912 & $\beta$-Ionone ${ }^{4}$ & - & & 1.19 & (16) & 1.01 & (8) & 15.77 & (7) & 8.42 & (15) & - & & 29.36 & (16) & 84.02 & (2) & 1.27 & (14) & - & \\
\hline 153 & 64.98 & 1933 & 1927 & Calamenene & - & & - & & - & & & & - & & - & & 4.86 & (4) & 3.73 & (4) & 2.14 & (16) & 1.98 & (2) \\
\hline 157 & 68.40 & 1994 & 2009 & Nerolidol $^{4}$ & 3.09 & (6) & 2.47 & (14) & - & & & & - & & - & & 2.81 & (12) & 1.96 & (3) & & & - & \\
\hline 158 & 69.19 & 2103 & 2089 & Elemol & - & & - & & 0.61 & (2) & 0.87 & (2) & 3.05 & (11) & 22.75 & (12) & 18.99 & (7) & 12.15 & (3) & - & & - & \\
\hline 159 & 71.74 & 2118 & 2113 & Cedrenol & 4.21 & (3) & 6.03 & (4) & 91.13 & (11) & 75.19 & (11) & - & & - & & 3.69 & (2) & 35.73 & (6) & 2.73 & (8) & 1.19 & (3) \\
\hline 162 & 73.21 & 2127 & 2129 & Spathulenol & 3.98 & (1) & 5.33 & (7) & 0.73 & (1) & 20.72 & (7) & & & - & & 19.20 & (14) & 1.13 & (13) & & & & \\
\hline & & & & $\begin{array}{c}\% \text { RPA } \\
\text { Volatile phenols }\end{array}$ & 32.87 & & 21.38 & & 17.69 & & 20.86 & & 53.03 & & 51.16 & & 16.77 & & 19.07 & & 24.06 & & 10.77 & \\
\hline 165 & 75.82 & 2142 & 2141 & $\begin{array}{l}\text { Eolatile phenois } \\
\text { Eugenol }^{4}\end{array}$ & 7.97 & (7) & 1.12 & (13) & 5.01 & (5) & 6.20 & (8) & 10.10 & (1) & 2.98 & (1) & 16.87 & (9) & 2.65 & (12) & 8.54 & (4) & 9.63 & (10) \\
\hline 167 & 76.56 & 2146 & 2170 & 4-Ethyl phenol ${ }^{4}$ & - & & 1.88 & (14) & - & & 1.21 & (2) & - & & 15.45 & (16) & - & & - & & - & & 1.16 & (12) \\
\hline & & & & $\%$ RPA & 0.61 & & 0.34 & & 0.17 & & 0.22 & & 0.33 & & 0.57 & & 0.63 & & 0.09 & & 0.28 & & 0.35 & \\
\hline
\end{tabular}


Table 1. Cont.

\begin{tabular}{|c|c|c|c|c|c|c|c|c|c|c|c|c|c|c|c|c|c|c|c|c|c|}
\hline \multirow{3}{*}{$\begin{array}{c}\text { Peak } \\
\mathbf{n}^{\circ}\end{array}$} & \multirow{3}{*}{$\underset{(\min )^{1}}{\mathbf{R T}}$} & \multirow{3}{*}{$\mathrm{KI}_{\mathrm{Cal}}{ }^{2}$} & \multirow{3}{*}{$\mathrm{KI}_{\mathrm{Lit}}{ }^{3}$} & \multirow{3}{*}{ Chemical Groups } & \multicolumn{17}{|c|}{ GC Peak Area $\left(\times 10^{6}\right)($ RSD) } \\
\hline & & & & & \multicolumn{2}{|c|}{ Grape } & \multicolumn{3}{|c|}{ Red Fruits } & \multicolumn{4}{|c|}{ Orange } & \multicolumn{4}{|c|}{ Pear } & \multicolumn{4}{|c|}{ Apple } \\
\hline & & & & & Fresh & Processed & \multicolumn{2}{|c|}{ Fresh } & Processed & \multicolumn{2}{|c|}{ Fresh } & \multicolumn{2}{|c|}{ Processed } & \multicolumn{2}{|c|}{ Fresh } & \multicolumn{2}{|c|}{ Processed } & \multicolumn{2}{|c|}{ Fresh } & \multicolumn{2}{|c|}{ Processed } \\
\hline & & & & $\begin{array}{l}\% \text { RPA } \\
\text { Ratty acids }\end{array}$ & 0.61 & 0.34 & 0.17 & & 0.22 & 0.33 & & 0.57 & & 0.63 & & 0.09 & & 0.28 & & 0.35 & \\
\hline 76 & 36.53 & 1447 & 1450 & Acetic acid ${ }^{4}$ & $34.60 \quad(5)$ & $20.77 \quad(6)$ & 1.37 & (15) & 7.08 & 2.45 & (12) & 32.50 & (5) & 7.74 & (11) & 13.49 & (8) & 24.06 & (3) & 12.43 & (6) \\
\hline 143 & 59.21 & 1815 & 1820 & Hexanoic acid 4 & 9.88 (15) & $2.40 \quad(16)$ & 26.48 & (13) & 18.33 & 68.43 & (13) & 58.96 & (2) & 1.26 & (5) & 2.98 & (3) & 8.16 & (11) & 1.03 & (13) \\
\hline \multirow[t]{2}{*}{160} & 72.60 & 2123 & 2083 & Octanoic acid 4 & $15.88 \quad(18)$ & 2.65 & 2.14 & (4) & 12.49 (13) & 2.73 & (14) & 14.74 & (8) & 2.60 & (10) & 2.07 & (10) & 11.71 & (4) & 5.36 & (7) \\
\hline & & & & $\begin{array}{l}\text { \% RPA } \\
\text { Furanic compounds }\end{array}$ & 4.58 & 2.93 & 1.01 & & 1.10 & 2.39 & & 3.30 & & 0.43 & & 0.65 & & 1.42 & & 0.62 & \\
\hline 77 & 37.12 & 1457 & 1455 & 2-Furfural ${ }^{4}$ & 3.39 & $159.04(4)$ & 0.79 & (7) & $194.85(7)$ & 4.67 & (6) & 124.55 & (10) & 1.25 & (1) & 247.37 & (4) & 3.26 & (5) & 197.05 & (4) \\
\hline \multirow[t]{2}{*}{93} & 43.71 & 1562 & 1560 & 5-Methyl-2-furfural ${ }^{4}$ & - & $2.40 \quad(6)$ & - & & $2.68 \quad(6)$ & - & & - & & - & & 2.73 & (13) & - & & - & \\
\hline & & & & $\% \mathrm{RPA}$ & 0.26 & 18.32 & 0.03 & & 5.75 & 0.15 & & 3.87 & & 0.05 & & 8.82 & & 0.11 & & 6.44 & \\
\hline
\end{tabular}

${ }^{1}$ RT: Retention time (min). ${ }^{2}$ Kovat index relative n-alkanes $\left(\mathrm{C}_{8}\right.$ to $\mathrm{C}_{20}$ ) on a BP-20 capillary column. ${ }^{3}$ Kovat index relative, reported in literature for equivalent capillary column [34,41-43]

${ }^{4}$ Volatile organic compounds (VOCs) identified by standard; -: Not detected; TDN: 1,2-dihydro-1,1,6-trimethylnaphtalene; Different superscript lowercase letters in a row indicate no significant differences between mean values at $p<0.05$, obtained by one-way ANOVA, followed by Tukey's test, for post-hoc multiple comparisons of means, for fresh and processed fruit juice $(n=2)$. 

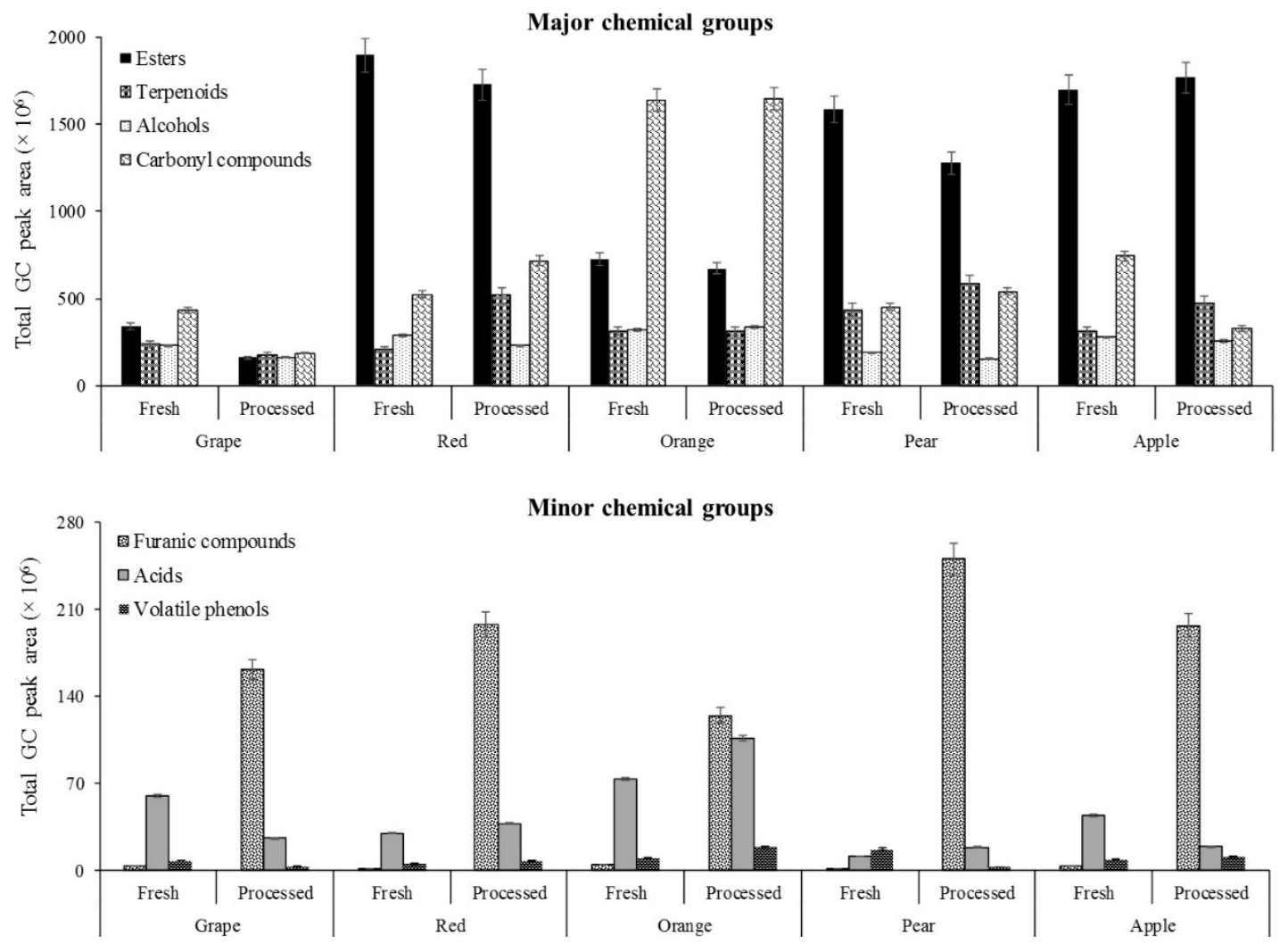

Figure 2. Total GC peak area of chemical groups identified in fresh and processed fruit juices.

In orange and grape juices, terpenoids was the most predominant chemical group, accounting for 53.0 and $32.9 \%$ of the total volatile composition of fresh juices, and 51.2 and $21.4 \%$ of that of processed juices, respectively. While the number of identified terpenoids in orange, pear, and apple juices was similar, its GC peak area in fresh and processed orange juices was three and four times higher than observed in fresh and processed juices from pear and apple, respectively. This trend is explained by the high GC peak areas of limonene, which were followed by those of germacrene D, geranyl acetone isomer and linalool, observed in orange juices (Table 1 and Table S2). On average, limonene represents 11.6 and $6.3 \%$ of the total volatile profile of fresh and processed juices, respectively.

On the other hand, the number of terpenoids identified in orange juice, independently of the type of juice, was two times higher than that in grape juice. The contribution of this chemical group to the total volatile profile of grape juice was two times higher than its contribution to that of red fruits, apple, and pear juices, irrespective of the type of juice (Table 1). Dihydrolinalool, followed by linalool and $\alpha$-terpineol, were the predominant terpenoids identified in grape juices (Table S2).

In the studied fresh juices, the contribution of alcohols to the total volatile profile ranged from 10.1 (orange) to $18.1 \%$ (grape), whereas in processed juices its contribution ranged from 9.7 (orange) to $20.7 \%$ (pear). The ethanol content was higher in the fresh juices from grape, orange, and pear than in the corresponding processed juices, whereas in the red fruit juices and apple juices, the GC peak area of ethanol was higher in the processed juices than in the fresh juices. 1-Propanol was the second most dominant alcohol in grapes and red fruit juices, whereas 3-methyl-1-butanol was the most dominant alcohol in orange and apple juices.

Regarding furanic compounds, a significant increase in terms of the total GC peak area (Figure S3) and \% RPA (Table 1) was observed for all investigated processed juices, unlike the fresh juices. The contribution of this chemical group to the total volatile profile of processed juices ranged from 3.9 (orange) to $18.3 \%$ (grape), whereas in fresh juices, its content was significantly low $(p<0.05)$, ranging from 0.03 (red fruits) to $0.3 \%$ (grapes). 


\subsection{Multivariate Data Analysis}

The data matrix (variables versus fresh and processed fruit juices) was normalized using cubic root and data scaling by the mean-center and was submitted to principal component analysis (PCA) to explore the main sources of similarity and variability as well as to characterize and differentiate the target types of fruit juices, fresh versus processed, based on the volatomic signature (Figure 3).

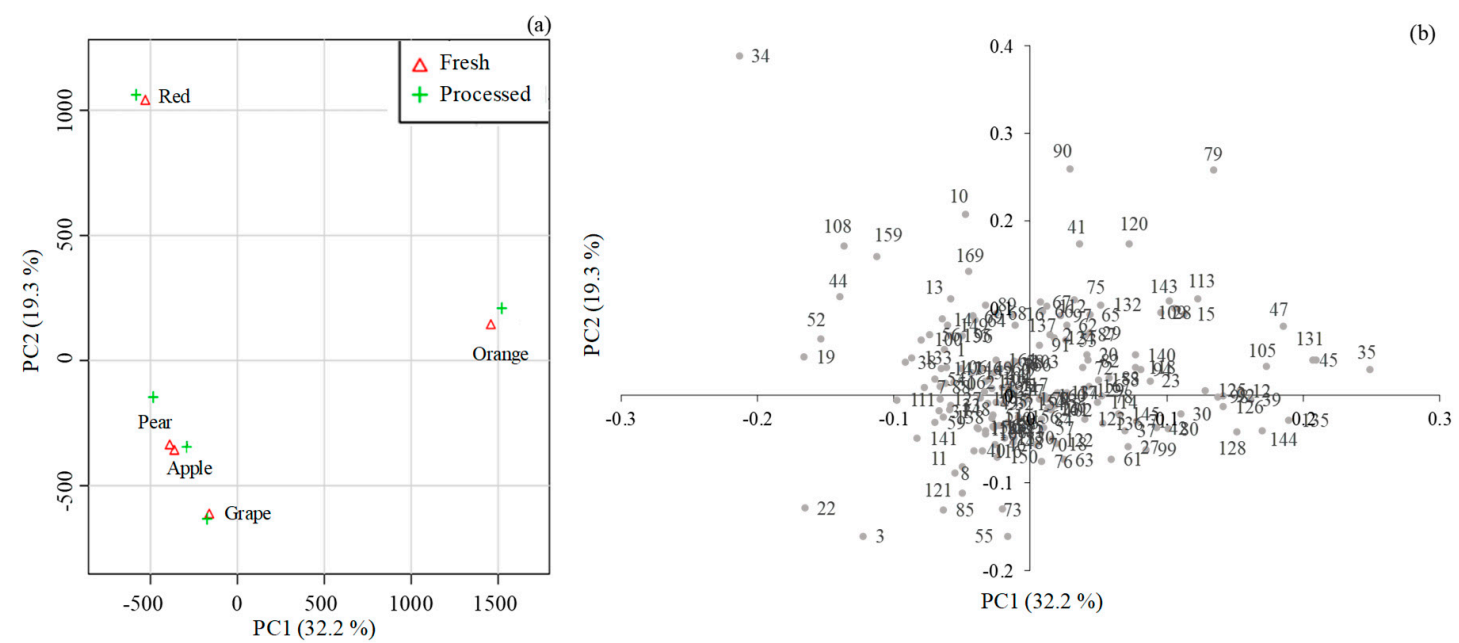

Figure 3. Principal component analysis (PCA) of the volatile signature of fresh and processed fruit juices. (a) PC1 $\times$ PC2 score scatter plot and (b) loading weight plot (attribution of the peak number is shown in Table S1).

For all of the analyzed fruit juices, the PCA score scatter plot of the two first principal components (PC1 (32.2\%) and PC2 (19.3\%)), which explain 51.5\% of the total variability of the GC-qMS dataset, is shown in Figure $3 \mathrm{a}$, and the corresponding loading weight plot, establishing the magnitude of each VOC (variable), is illustrated in Figure 3b. Analysis of the loading plot revealed the VOCs responsible for the discrimination of fruit juices based on fruit species/type. Fresh and processed red fruit juices, projected in PC1 negative and PC2 positive, were mainly characterized by methyl hexanoate (34), whereas apple, pear, and grape juices (PC1 and PC2 negative) were mainly characterized by ethyl acetate (3) and 3-methylbutanol acetate (22). Finally, orange juices projected in PC1 and PC2 positive were mainly associated with limonene (35).

Additionally, the partial least squares-discriminant analysis (PLS-DA) (Figure 4a,b) was used as a supervised cluster, and 30 differently expressed VOCs were found with variable importance in projection (VIP) scores higher than 1: 2-furfural (77), ethyl dodecanoate (146), valencene (123), ethyl 3-hydrohexanoate (112), ethyl 3-methylbutanoate (18), methyl hexanoate (34), hexyl acetate (44), 2,4-decadienal (127), linalool (90), and propyl butanoate (40) were the most significant. Figure S3 shows the resulting dendrogram associated with the heat map constructed using Pearson's correlation, providing an intuitive visualization of the dataset, which is often applied to identify samples or features that are unusually high or low. Five major clusters were responsible for grouping the juice samples by fruit variety (horizontal axis), and not by the type of juice (fresh or processed). An analogous color tone in the heat map indicated the area, a group of samples, considering that the concentration of the analyzed VOCs was similar.

Figure 5 shows the heat map constructed using Pearson's correlation for the VOCs with VIP scores higher than 1. It is possible to observe that 2-furfural (77), ethyl dodecanoate (146), ethyl 3-hydrohexanoate (112), ethyl 3-methylbutanoate (18), 4-ethy phenol (167), butyl octanoate (104), 2-heptanone (32), and $\gamma$-decalactone (164) had a higher chromatographic area in processed juice than in fresh fruit juice, whereas valencene (123) had a higher chromatographic area in fresh than in processed fruit juice. 

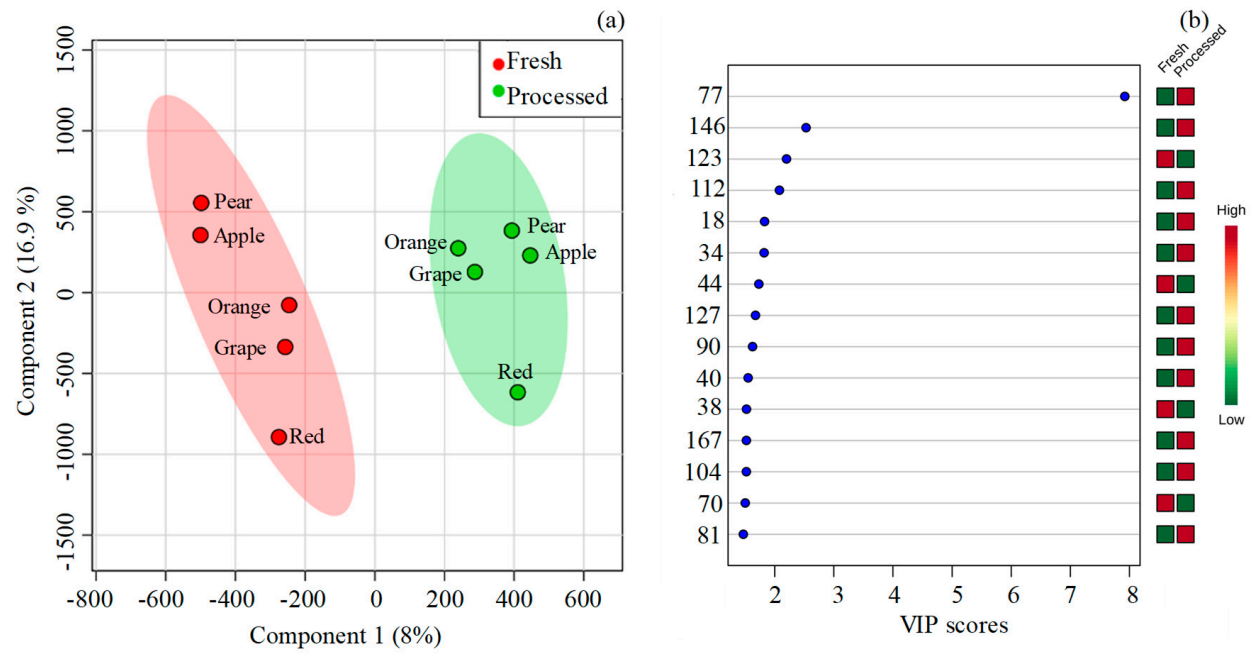

Figure 4. Partial least squares-discriminant analysis (PLS-DA) of the volatile signature of fresh and processed fruit juices. (a) Score scatter plot, and (b) Variable importance in projection (VIP) scores (attribution of the peak number is shown in Table 1).

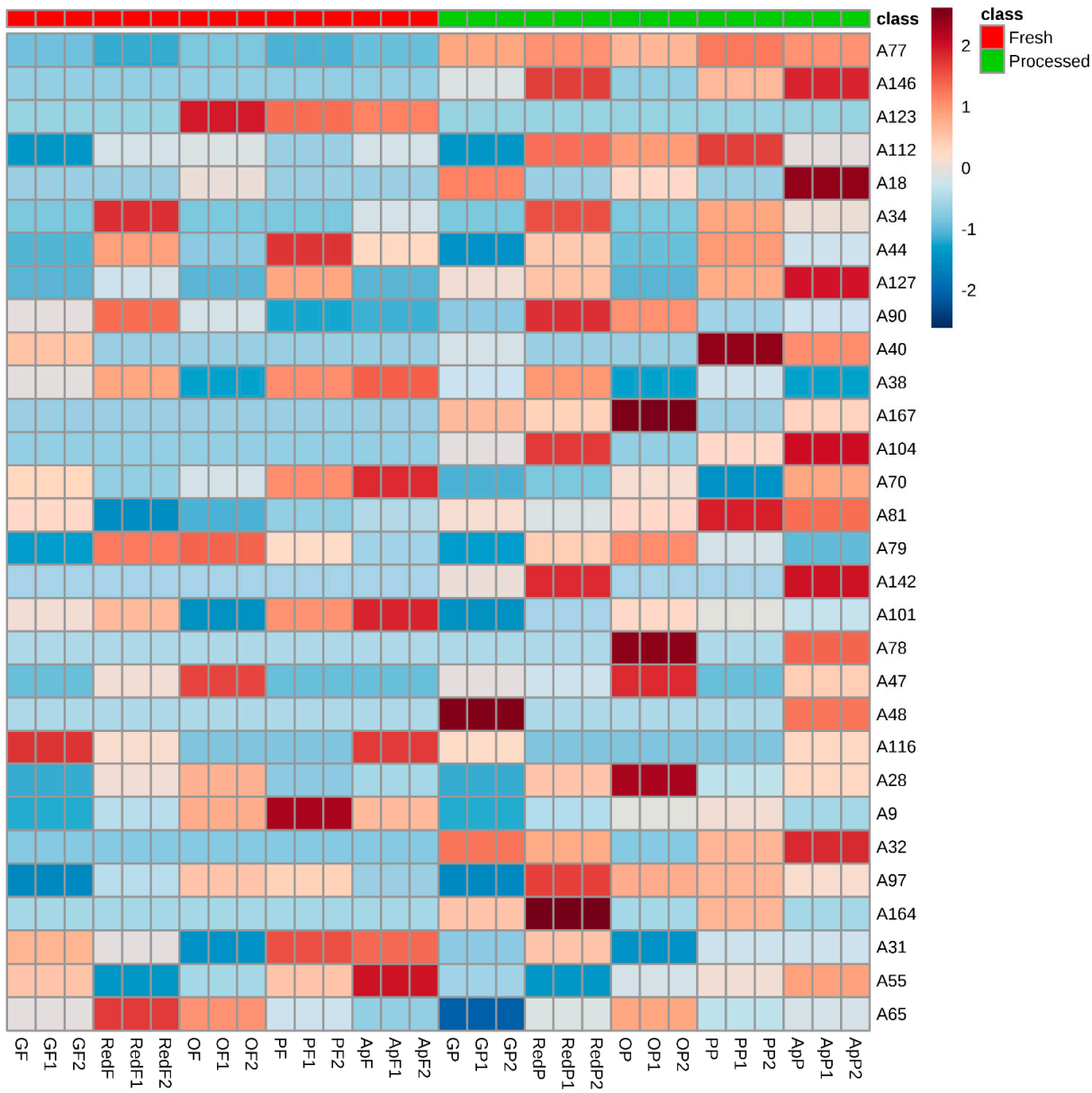

Figure 5. Hierarchical cluster analysis (HCA). The heat maps of the 30 VOCs (VIP values > 1), identified in fresh and processed fruit juices were generated by an average algorithm and Pearson's distance analysis (attribution of the peak number is shown in Table 1). Abbreviation: OP-orange juice processed, $\mathrm{OF}$ - orange juice fresh, GP — grape juice processed, GF-grape juice fresh, $\mathrm{PP}$ - pear juice processed, $\mathrm{PF}$ - pear juice fresh, $\mathrm{ApP}$ —apple juice processed, $\mathrm{ApF}$ - apple juice fresh. 


\section{Discussion}

In sum, fruit aroma is an important indicator, which may reflect the quality of future fruit juice. The contribution of each VOC to the specific aroma signature of each fruit species/types depends on the activity and substrate specificity of the relevant enzymes in the biosynthetic pathway, the substrate availability, the OT above which VOC can be detected by smell, and the presence of the other VOCs [5]. The esters represented more than $45.15 \%$ of the total volatile profile of apple, pear, and red fruit juices, which is in accordance with previous studies that report esters as the most abundant chemical group in pear and apple fruit juices $[6,7,11,13]$. Regarding this chemical group, hexyl acetate, a dominant esters in pear juice, has been reported as possessing a strong pear-like odor [12]. Moreover, ethyl octanoate contributes to sweet and fruit odors in pears, while hexanal, ethyl acetate, hexyl acetate, ethyl butanoate, and butanol are identified as impact volatiles in Conference pears [13]. On the other hand, terpenoids, one of the most dominant chemical groups of VOCs found in orange and grape fruit juices, have shown beneficial functions as well as nutraceutical activities. Recently, some studies in the field of cancer research and food nutrition sciences have been performed on these VOCs due to their potential anticancer properties [44]. Among them, limonene (a dominant VOC in orange juice) has been reported to be a bioactive food component from citrus fruits, with a potential role in breast cancer prevention and treatment [45]. In addition, limonene reduces the oxidative stress in Streptozotocin-induced diabetic rats by decreasing lipid peroxidation and sparing the activities of antioxidant enzymes [46]. Linalool (a dominant VOC in red fruit juice) and terpineol (a dominant in grape fruit juice) are responsible for the fruity and floral notes of food matrices. Moreover, linalool and its degradation product, $\alpha$-terpineol, have been reported as potent antimicrobial agents against periodontopathic and cariogenic bacteria [47]. Anti-inflammatory effects were also associated with these terpenes [48]. $\alpha$-Farnesene, a specific terpenoid, with green and herbaceous aroma descriptors, has been reported as the most abundant terpene in fresh apple juice [49], which is in agreement with the results of our study, since we detected GC peak areas $\left(\times 10^{6}\right)$ in fresh and processed apple juices of 419.32 and 8.16, respectively. Moreover, according to previous studies, the aroma of squeezed orange juice is primarily associated to esters (e.g., ethyl butanoate, ethyl 2-methylpropanoate), aldehydes (e.g., acetaldehyde, hexanal, octanal), in addition to a smaller number of terpenoids (e.g., myrcene, $\alpha$-pinene, potentially limonene, linalool, geranial). All these VOCs are responsible for the citrus, green, sweet fruit, and floral notes from fresh orange juice [50-52].

There are differences between the VOCs contained in fresh and processed fruit juices, with processed juices having the highest levels of VOCs. Indeed, a recent study of fresh and commercial pomegranate juice indicated that there were marked differences between endogenous VOCs extracted in the laboratory versus what was often found in commercial products [53]. This fact may be due to several characteristics of fruits (e.g., geographical and botanical origin, varieties, ripening stage, farming practices, and post-harvest handling), the process applied in juice extraction and its production (e.g., enzymes, heat, and filtration), as well as storage conditions [5]. A recent assay reported that the volatile loss generally continued through pasteurized storage, and the lowest volatile levels were recovered after one, two, and four months of storage [54]. Another study highlighted that changes in volatile chemicals could provide useful information about juice processing for the selection of the optimal harvest time and for setting a precise blending strategy [55]. Our study showed that the volatile profile of processed juices is more complex than that of fresh juices. This result is in accordance with a previous work carried out by Schmutzer and colleagues [49], in which fresh apple juice was compared with commercial apple juice. These authors highlighted that the differentiation criteria between fresh and commercial juices based on individual compounds implied a difficult characterization process, which is not helpful in establishing a quality index. Consequently, the most appropriate and useful method is to compare groups of VOCs (in our study: esters, alcohols, carbonyl compounds, terpenoids, volatile phenols, acids, and furanic compounds). In general, fresh juices were mainly characterized by the presence of esters and terpenoids, while furanic compounds (Maillard products) were important contributors to the profile of processed juices. Our findings are in accordance 
with a previous report concerning pomegranate juices [56]. Moreover, storage time and temperature can also change juice aroma profiles due to Maillard, strecker, and acid catalyzed hydration reactions. The formation of methional from methionine is probably the most significant example of Strecker degradation in orange juice [51]. Methional concentration in processed orange juice is higher than fresh juice (Table 1). Moreover, esters as stated above represent an important contribution to fruit juice odor, and the level of total esters in aqueous essence has been used as a quality index [51]. Nevertheless, their concentration in processed juice was lower than fresh juice, since during processing many esters rapidly hydrolyzed by hydrolase enzymes.

The presence of ethanol in the investigated fruit juices is indicative of the fermentation process caused by microorganisms as a consequence of inefficient sterilization of raw materials or later contamination. A previous study reported that high ethanol contents were observed mainly in juices without preservatives, particularly freshly-squeezed juices. Octanal and decanal, the most dominant compounds, of the carbonyl group found, in orange juice unlike other juices, have been considered to be important contributors to the orange flavor and standard of orange peel oil [57]. An important point to highlight is that the production of excessive volatile phenols is regarded as spoilage. In our study, 4-ethyl phenol, in particular, was only detected in processed juices, suggesting a possible microbial contamination (e.g., Brettanomyces sp). It has been reported [58] that this phenol may provide woody, smoky, leather, and animal off-odors. In the same sense, Daud and colleagues [59] reported that another family of compounds, which may contribute to undesirable smells or odors, is acidic compounds such as octanoic and hexanoic acids.

The data obtained in the current study represent an added value to the existing knowledge, as well as provide a useful platform to improve the organoleptic characteristics of the processed juices, and consequently contribute to their economic valorization and improve their acceptance by consumers.

\section{Materials and Methods}

\subsection{Material and Chemicals}

All chemicals were of analytical quality. Sodium chloride ( $\mathrm{NaCl}, 99.5 \%)$ was supplied from Panreac (Spain, Barcelona) and the VOC standards used for identification were purchased from Sigma-Aldrich (Madrid, Spain) and Acros Organics (Geel, Belgium), with a purity $\geq 98 \%$. Helium of purity 5.0 (Air Liquide, Lisbon, Portugal) was utilized as the GC carrier gas. The glass vials, SPME fibers and SPME holder for manual sampling were purchased from Supelco (Bellefonte, PA, USA). The KI was calculated through the injection of a series of $C_{8}$ to $C_{20}$ straight-chain n-alkanes (concentration of $40 \mathrm{mg} / \mathrm{L}$ in $n$-hexane), supplied by Fluka (Buchs, Switzerland).

\subsection{Fruit Juice Samples}

Healthy mature-state and undamaged fruit samples from fresh grapes (Vitis vinifera L.), wild red fruits (Fragaria x ananassa Duch. Vaccinium corymbosum L., Rubus idaeus L.), orange (Citrus sinensis L.), pear (Pyrus communis L.), and apple (Malus domestica Borkh.) were collected at maturity state based on the sugar/acid ratio, color, and $\mathrm{pH}$ from producers in the southwest region of Madeira Island, Portugal during the 2016 harvest and were immediately taken to the process line at $4{ }^{\circ} \mathrm{C}$. For each sampling, fruits were picked randomly throughout the experimental vineyard, taking into consideration the balance between shadow and sun exposure in the different vineyard locations, following a z-shaped pattern to avoid edge and center effects. In the process line, the fresh fruits were screened, inspected, washed, mechanically crushed, and submitted to an enzymatic treatment using pectinase enzyme (concentration of enzyme: $5 \mathrm{mg} / 100 \mathrm{~g}$; incubation time: $2 \mathrm{~h}$; and temperature: $35^{\circ} \mathrm{C}$ ). Operations such as washing, sorting and inspecting require attention to mass and heat transfer. Cooling depends upon heat transfer from fruit to air (possibly water). Cleaning sometimes involved the physical removal of surface debris by brushes which reduced the number of microbes. Inspecting sometimes involved the 
removal of raw materials which did not meet the standards of the quality of analysis. The enzymatic activity was monitored by adding five milliliters of juice to $15 \mathrm{~mL}$ of $\mathrm{HCl}$-acidified ethyl alcohol, observing the mixture for $5 \mathrm{~min}$ for gel formation. No gel formation meant that the depectinization was complete. After this, the juice was extracted at room temperature $\left(25^{\circ} \mathrm{C}\right)$, using the equipment appropriate for each fruit variety, and then filtrated (XP-201 polyvinylidene fluoride membrane) under vacuum. At this stage, fresh fruit juices were taken, in $250-\mathrm{mL}$ amber glass vials and immediately transported to the laboratory at $4{ }^{\circ} \mathrm{C}$, aliquoted in 20-mL amber glass vials and frozen at $-80{ }^{\circ} \mathrm{C}$ until analysis. The remaining fruit juice was submitted to a pasteurization process at $80-95^{\circ} \mathrm{C}$ for 1 to $10 \mathrm{~min}$ in intermediate tanks to eliminate bacteria without changing the fresh flavor of the fruit juice. After this process, the fruit juice was submitted to a cooling batch $\left(4\right.$ to $\left.5{ }^{\circ} \mathrm{C}\right)$ and sterile storage. At this stage, the processed fruit juice was taken, transported to the laboratory, aliquoted, and frozen at $-80{ }^{\circ} \mathrm{C}$ until analysis. All analyses were performed in triplicate.

\subsection{Headspace Solid-Phase Microextraction}

The HS-SPME experimental parameters used in this work were previously optimized in several works, developed in our group $[15,19,41,60]$. Briefly, aliquots of $5 \mathrm{~mL}$ of juice samples were placed into a 10-mL glass vial. After the addition of $10 \%(w / v)$ of $\mathrm{NaCl}$ and stirring $(0.5 \times 0.1 \mathrm{~mm}$ bar) at $400 \mathrm{rpm}$, the vial was capped with a PTFE (polytetrafluoroethylene) septum and an aluminum cap (Chromacol, Hertfordshire, UK). The vial was placed in a thermostated bath, adjusted at $40.0 \pm 0.1^{\circ} \mathrm{C}$ for $5 \mathrm{~min}$, and then the 50/30 $\mu \mathrm{m}$ divinylbenzene/carboxen/polydimethylsiloxane (DVB/CAR/PDMS) fiber was inserted into the headspace for $45 \mathrm{~min}$. Each sample was analyzed in triplicate. Blanks, corresponding to the analysis of the coating fiber and not submitted to any extraction procedure, were run between sets of three analyses.

\subsection{Gas Chromatography-Mass Spectrometry Conditions}

The gas chromatography mass spectrometry conditions were previously established $[15,41]$. The fiber of the SPME containing the VOCs was manually introduced into the GC injection port at $250{ }^{\circ} \mathrm{C}$ (equipped with a glass liner, $0.75 \mathrm{~mm}$ I.D.) for $5 \mathrm{~min}$ for desorption. The desorbed VOCs were analyzed in an Agilent Technologies 6890N Network gas chromatography equipped with a BP-20 fused silica capillary column $(30 \mathrm{~m} \times 0.25 \mathrm{~mm}$ I.D. $\times 0.25-\mu \mathrm{m}$ film thickness $)$ supplied by SGE (Darmstadt, Germany), connected to an Agilent 5973N quadrupole mass selective detector. Helium (Air Liquid, Lisbon, Portugal) was used as the carrier gas at a flow rate of $1.0 \mathrm{~mL} / \mathrm{min}$ (column-head pressure: $12 \mathrm{psi}$ ). The injections were performed in the splitless mode (5 $\mathrm{min})$. The GC oven temperature was programmed as follows: $45^{\circ} \mathrm{C}(1 \mathrm{~min})$, then increased by $2{ }^{\circ} \mathrm{C} / \mathrm{min}$ to $100{ }^{\circ} \mathrm{C}$ (3 min), $5{ }^{\circ} \mathrm{C} / \mathrm{min}$ to $130{ }^{\circ} \mathrm{C}(5 \mathrm{~min})$, and finally $20^{\circ} \mathrm{C} / \mathrm{min}$ to $220^{\circ} \mathrm{C}(2 \mathrm{~min})$. For the MS system, the temperatures of the transfer line, quadrupole, and ionization source were 250,150 , and $230^{\circ} \mathrm{C}$, respectively. The electron impact mass spectra were recorded at $70 \mathrm{eV}$, and the ionization current was about $30 \mu \mathrm{A}$. The acquisitions were performed in full scan mode $(30-300 \mathrm{~m} / \mathrm{z})$. The identification of the VOCs was achieved by (i) comparing the GC retention times and mass spectra, with those, when available, of the standard compounds; (ii) comparing all mass spectra with the data system library (NIST, 2005 software, Mass Spectral Search Program V.2.0d, Agilent, Washington, USA); and (iii) determining the kovat index (KI) values, according to the van den Dool and Kratz equation [61]. For the KI determination, a $C_{8}-C_{20}$ n-alkanes series was used, and the values were compared, when available, with values reported in the literature for similar columns [34,41-43,62].

\subsection{Statistical Analysis}

The obtained data were analyzed with Metaboanalyst 4.0 [63], which included data pre-processing to remove VOCs with missing values (MVs), MVs imputation of the resulting data (by Bayesian principal component analysis, PCA method), and normalization (data transformation by cubic root and data scaling by the mean-center). The normalized data were further subjected to one-way ANOVA, 
followed by Tukey's test $(p<0.05)$, for post-hoc multiple comparisons of means and multivariate statistical analysis, namely, principal component analysis (PCA) and partial least squares-discriminant analysis (PLS-DA). This provided insights into the separations between the fresh and processed fruit juices and allowed VOCs that may have indicated differences among the samples sets to be identified. Finally, Pearson's correlation was used to build the heat map of the fresh and processed fruit juices.

\section{Conclusions}

The HS-SPME/GC-qMS has proven to be a suitable and solvent-free approach to the establishment of the volatile signature of fresh and processed juices from different fruits (pear, orange, red fruits, grape, and apple). This analytical technique, combined with multivariate analysis, may offer to fruit juice industries an alternative technique for routine analysis in the monitoring of processes that may affect aroma compounds in order to obtain fruit juices with high-quality flavors. For processed juices, special attention should be paid during the production process in order to avoid possible deleterious effects associated with the formation of furanic compounds, since a significant increase was observed from fresh to processed fruit juices of the same batch. Storage time and temperature can also change juice aroma profiles due to Maillard, Strecker, and acid catalyzed hydration reactions. In this sense, methional is probably the most significant example of Strecker degradation in orange juice, and it was possible to observe that its concentration in processed fruit juice was higher than in fresh fruit juice. On the other hand, esters contribute positively to fruit juice odor, with fruit and floral notes. However, their relative concentration in processed fruit juice was lower than fresh fruit juice, since during processing many esters rapidly hydrolyzed by hydrolase enzymes. Consequently, it was expected that their contribution to volatile profile on processed fruit juice was lower. These findings represent a suitable tool for providing data on fruit markers, which may be useful in defining the production procedures (e.g., enzyme, filtration, heat treatment, pasteurization, packing or storage), improving the organoleptic characteristics of the corresponding juices, and consequently contributing to the economic valorization and consumer acceptance of juices. Moreover, since the data obtained in the study correspond to one harvest, more studies need be conducted to investigate the typicality and reproducibility of the patterns of VOCs, across multiple harvests, and geographical and agronomic conditions.

Supplementary Materials: The following are available online at http:/ /www.mdpi.com/1420-3049/24/5/974/s1, Figure S1: Total ion chromatograms obtained by HS-SPME $E_{D V B} / C A R / P D M S / G C-q M S$ analysis of processed fruit juices (for identification of peak numbers see Table 1), Figure S2: Overload of GC-qMS chromatograms (enlarged part of the chromatograms of Figure 1) showing the comparison of the typical profile of 2-furfural identified in fresh and processed fruit juices, Figure S3: Hierarchical cluster analysis (HCA). The heat maps of the 169 VOCs identified in fresh and processed fruit juices were generated by average algorithm and Pearson distance analysis (attribution of the peak number is shown in Table 1). Abbreviation: OP-orange juice processed, OF-orange juice fresh, GP-grape juice processed, GF-grape juice fresh, $\mathrm{PP}$ - pear juice processed, PF-pear juice fresh, $\mathrm{ApP}$ - apple juice processed, ApF-apple juice fresh, Table S1: Common volatile organic compounds (VOCs) identified in fresh and processed fruit juices, Table S2: Predominant VOCs in fresh and processed fruit juices analyzed using HS-SPME/GC-MS methodology.

Author Contributions: R.P., C.S. and P.S. performed the design of experiments, data and statistical analysis; R.P. and S.M. performed manuscript preparation; R.P. and J.S.C. performed the conception of study, manuscript preparation, and editing.

Funding: This research received no external funding.

Acknowledgments: This work was supported by the FCT-Fundação para a Ciência e a Tecnologia (project PEst-OE/QUI/UI0674/2013, CQM, Portuguese Government funds), Madeira 14-20 Program (project PROEQUIPRAM-Reforço do Investimento em Equipamentos e Infraestruturas Científicas na RAM-M1420-01-0145-FEDER-000008) and by ARDITI-Agência Regional para o Desenvolvimento da Investigação Tecnologia e Inovação through the project M1420-01-0145-FEDER-000005-Centro de Química da Madeira-CQM+ (Madeira 14-20). The authors also acknowledge Post-Doc grants, SFRH/BPD/97387/2013 and ARDITI-CQM/2017/008-PDG to Rosa Perestrelo and Sonia Medina, respectively, and PhD grants to Catarina Silva (SFRH/BD/97039/2013) and Pedro Silva (ARDITI; M1420 Project-09-5369-FSE-000001).

Conflicts of Interest: The authors declare no conflict of interest. 


\section{References}

1. Slavin, J.L.; Lloyd, B. Health benefits of fruits and vegetables. Adv. Nutr. An Int. Rev. J. 2012, 3, 506-516. [CrossRef] [PubMed]

2. Wang, X.; Ouyang, Y.; Liu, J.; Zhu, M.; Zhao, G.; Bao, W.; Hu, F.B. Fruit and vegetable consumption and mortality from all causes, cardiovascular disease, and cancer: Systematic review and dose-response meta-analysis of prospective cohort studies. BMJ (Clin. Res. Ed.) 2014, 349, g4490. [CrossRef] [PubMed]

3. D'Agostino, M.F.; Sanz, J.; Sanz, M.L.; Giuffrè, A.M.; Sicari, V.; Soria, A.C. Optimization of a solid-phase microextraction method for the gas chromatography-mass spectrometry analysis of blackberry (Rubus ulmifolius Schott) fruit volatiles. Food Chem. 2015, 178, 10-17. [CrossRef] [PubMed]

4. Khalil, M.N.A.; Fekry, M.I.; Farag, M.A. Metabolome based volatiles profiling in 13 date palm fruit varieties from Egypt via SPME GC-MS and chemometrics. Food Chem. 2017, 217, 171-181. [CrossRef] [PubMed]

5. El Hadi, M.; Zhang, F.-J.; Wu, F.-F.; Zhou, C.-H.; Tao, J. Advances in fruit aroma volatile research. Molecules 2013, 18, 8200-8229. [CrossRef] [PubMed]

6. Ferreira, L.; Perestrelo, R.; Caldeira, M.; Câmara, J.S. Characterization of volatile substances in apples from Rosaceae family by headspace solid-phase microextraction followed by GC-qMS. J. Sep. Sci. 2009, 32, 1875-1888. [CrossRef] [PubMed]

7. Holland, D.; Larkov, O.; Bar-Ya'akov, I.; Bar, E.; Zax, A.; Brandeis, E.; Ravid, U.; Lewinsohn, E. Developmental and Varietal Differences in Volatile Ester Formation and Acetyl-CoA: Alcohol Acetyl Transferase Activities in Apple (Malus domestica Borkh.). Fruit 2005. [CrossRef]

8. Rizzolo, A.; Grassi, M.; Eccher Zerbini, P. Influence of harvest date on ripening and volatile compounds in the scab-resistant apple cultivar 'Golden Orange'. J. Hortic. Sci. Biotechnol. 2006, 81, 681-690. [CrossRef]

9. Villatoro, C.; Altisent, R.; Echeverría, G.; Graell, J.; López, M.L.; Lara, I. Changes in biosynthesis of aroma volatile compounds during on-tree maturation of 'Pink Lady ${ }^{\circledR}$ ' apples. Postharvest Biol. Technol. 2008, 47, 286-295. [CrossRef]

10. Lara, I.; Miró, R.M.; Fuentes, T.; Sayez, G.; Graell, J.; López, M.L. Biosynthesis of volatile aroma compounds in pear fruit stored under long-term controlled-atmosphere conditions. Postharvest Biol. Technol. 2003, 29, 29-39. [CrossRef]

11. Kahle, K.; Preston, C.; Richling, E.; Heckel, F.; Schreier, P. On-line gas chromatography combustion/pyrolysis isotope ratio mass spectrometry (HRGC-C/P-IRMS) of major volatiles from pear fruit (Pyrus communis) and pear products. Food Chem. 2005, 91, 449-455. [CrossRef]

12. Rapparini, F.; Predieri, S. Pear Fruit Volatiles. In Horticultural Reviews; John Wiley \& Sons, Inc.: Oxford, UK, 2010; pp. 237-324.

13. Rizzolo, A.; Cambiaghi, P.; Grassi, M.; Zerbini, P.E. Influence of 1-Methylcyclopropene and Storage Atmosphere on Changes in Volatile Compounds and Fruit Quality of Conference Pears. J. Agric. Food Chem. 2005, 53, 9781-9789. [CrossRef] [PubMed]

14. Kelebek, H.; Selli, S. Determination of volatile, phenolic, organic acid and sugar components in a Turkish cv. Dortyol (Citrus sinensis L. Osbeck) orange juice. J. Sci. Food Agric. 2011, 91, 1855-1862. [CrossRef] [PubMed]

15. Perestrelo, R.; Barros, A.S.; Rocha, S.M.; Câmara, J.S. Optimisation of solid-phase microextraction combined with gas chromatography-mass spectrometry based methodology to establish the global volatile signature in pulp and skin of Vitis vinifera L. grape varieties. Talanta 2011, 85, 1483-1493. [CrossRef] [PubMed]

16. Bellincontro, A.; Nicoletti, I.; Valentini, M.; Tomas, A.; De Santis, D.; Corradini, D.; Mencarelli, F. Integration of Nondestructive Techniques with Destructive Analyses to Study Postharvest Water Stress of Winegrapes. Am. J. Enol. Vitic. 2009, 60, 57-65.

17. Fenoll, J.; Manso, A.; Hellín, P.; Ruiz, L.; Flores, P. Changes in the aromatic composition of the Vitis vinifera grape Muscat Hamburg during ripening. Food Chem. 2009, 114, 420-428. [CrossRef]

18. Perestrelo, R.; Silva, C.; Silva, P.; Câmara, J.S. Unraveling Vitis vinifera L. grape maturity markers based on integration of terpenic pattern and chemometric methods. Microchem. J. 2018, 142, 367-376. [CrossRef]

19. Porto-Figueira, P.; Freitas, A.; Cruz, C.J.; Figueira, J.; Câmara, J.S. Profiling of passion fruit volatiles: An effective tool to discriminate between species and varieties. Food Res. Int. 2015, 77, 408-418. [CrossRef]

20. Riu-Aumatell, M.; Castellari, M.; López-Tamames, E.; Galassi, S.; Buxaderas, S. Characterisation of volatile compounds of fruit juices and nectars by HS/SPME and GC/MS. Food Chem. 2004, 87, 627-637. [CrossRef] 
21. Alves Filho, E.G.; Rodrigues, T.H.S.; Fernandes, F.A.N.; de Brito, E.S.; Cullen, P.J.; Frias, J.M.; Bourke, P.; Cavalcante, R.S.; Almeida, F.D.L.; Rodrigues, S. An untargeted chemometric evaluation of plasma and ozone processing effect on volatile compounds in orange juice. Innov. Food Sci. Emerg. Technol. 2017. [CrossRef]

22. Evelyn; Kim, H.J.; Silva, F.V.M. Modeling the inactivation of Neosartorya fischeri ascospores in apple juice by high pressure, power ultrasound and thermal processing. Food Control 2016, 59, 530-537. [CrossRef]

23. Yi, J.; Kebede, B.T.; Hai Dang, D.N.; Buvé, C.; Grauwet, T.; Van Loey, A.; Hu, X.; Hendrickx, M. Quality change during high pressure processing and thermal processing of cloudy apple juice. LWT 2017, 75, 85-92. [CrossRef]

24. Kebede, B.; Lee, P.Y.; Leong, S.Y.; Kethireddy, V.; Ma, Q.; Aganovic, K.; Eyres, G.T.; Hamid, N.; Oey, I. A Chemometrics Approach Comparing Volatile Changes during the Shelf Life of Apple Juice Processed by Pulsed Electric Fields, High Pressure and Thermal Pasteurization. Foods 2018, 7, 169. [CrossRef] [PubMed]

25. Gao, G.; Zhao, L.; Ma, Y.; Wang, Y.; Sun, Z.; Liao, X. Microorganisms and Some Quality of Red Grapefruit Juice Affected by High Pressure Processing and High Temperature Short Time. Food Bioprocess Technol. 2015, 8, 2096-2108. [CrossRef]

26. Evelyn; Silva, F.V.M. High pressure processing pretreatment enhanced the thermosonication inactivation of Alicyclobacillus acidoterrestris spores in orange juice. Food Control 2016, 62, 365-372. [CrossRef]

27. Mastello, R.B.; Janzantti, N.S.; Bisconsin-Júnior, A.; Monteiro, M. Impact of HHP processing on volatile profile and sensory acceptance of Pêra-Rio orange juice. Innov. Food Sci. Emerg. Technol. 2018, 45, 106-114. [CrossRef]

28. Yuk, H.G.; Sampedro, F.; Fan, X.; Geveke, D.J. Nonthermal Processing of Orange Juice Using a Pilot-Plant Scale Supercritical Carbon Dioxide System with a Gas-Liquid Metal Contactor. J. Food Process. Preserv. 2014, 38, 630-638. [CrossRef]

29. Marszałek, K.; Woźniak, Ł.; Barba, F.J.; Skapska, S.; Lorenzo, J.M.; Zambon, A.; Spilimbergo, S. Enzymatic, physicochemical, nutritional and phytochemical profile changes of apple (Golden Delicious L.) juice under supercritical carbon dioxide and long-term cold storage. Food Chem. 2018, 268, 279-286. [CrossRef] [PubMed]

30. Amaral, G.V.; Silva, E.K.; Costa, A.L.R.; Alvarenga, V.O.; Cavalcanti, R.N.; Esmerino, E.A.; Guimarães, J.T.; Freitas, M.Q.; Sant'Ana, A.S.; Cunha, R.L.; et al. Whey-grape juice drink processed by supercritical carbon dioxide technology: Physical properties and sensory acceptance. LWT 2018, 92, 80-86. [CrossRef]

31. Silva, P.; Freitas, J.; Silva, C.L.C.L.; Perestrelo, R.; Nunes, F.M.F.M.; Câmara, J.S.J.S. Establishment of authenticity and typicality of sugarcane honey based on volatile profile and multivariate analysis. Food Control 2016, 73, 1176-1188. [CrossRef]

32. Ivanova, V.; Stefova, M.; Vojnoski, B.; Stafilov, T.; Bíró, I.; Bufa, A.; Felinger, A.; Kilár, F. Volatile composition of Macedonian and Hungarian wines assessed by GC/MS. Food Bioprocess Technol. 2013, 6, 1609-1617. [CrossRef]

33. Lee, S.-N.; Kim, N.-S.; Lee, D.-S. Comparative study of extraction techniques for determination of garlic flavor components by gas chromatography-mass spectrometry. Anal. Bioanal. Chem. 2003, 377, 749-756. [CrossRef] [PubMed]

34. Mendes, B.; Gonçalves, J.; Câmara, J.S. Effectiveness of high-throughput miniaturized sorbent- and solid phase microextraction techniques combined with gas chromatography-mass spectrometry analysis for a rapid screening of volatile and semi-volatile composition of wines-A comparative study. Talanta 2012, 88, 79-94. [CrossRef] [PubMed]

35. Belandria, V.; Aparecida de Oliveira, P.M.; Chartier, A.; Rabi, J.A.; de Oliveira, A.L.; Bostyn, S. Pressurized-fluid extraction of cafestol and kahweol diterpenes from green coffee. Innov. Food Sci. Emerg. Technol. 2016, 37, 145-152. [CrossRef]

36. Ochiai, N.; Sasamoto, K.; Takino, M.; Yamashita, S.; Daishima, S.; Heiden, A.C.; Hoffmann, A. Simultaneous determination of preservatives in beverages, vinegar, aqueous sauces, and quasi-drug drinks by stir-bar sorptive extraction (SBSE) and thermal desorption GC-MS. Anal. Bioanal. Chem. 2002, 373, 56-63. [CrossRef] [PubMed]

37. Uekane, T.M.; Nicolotti, L.; Griglione, A.; Bizzo, H.R.; Rubiolo, P.; Bicchi, C.; Rocha-Leão, M.H.M.; Rezende, C.M. Studies on the volatile fraction composition of three native Amazonian-Brazilian fruits: Murici (Byrsonima crassifolia L., Malpighiaceae), bacuri (Platonia insignis M., Clusiaceae), and sapodilla (Manilkara sapota L., Sapotaceae). Food Chem. 2017, 219, 13-22. [CrossRef] [PubMed] 
38. Sentandreu, E.; Cerdán-Calero, M.; Sendra, J.M. Phenolic profile characterization of pomegranate (Punica granatum) juice by high-performance liquid chromatography with diode array detection coupled to an electrospray ion trap mass analyzer. J. Food Compos. Anal. 2013, 30, 32-40. [CrossRef]

39. Tempere, S.; Schaaper, M.H.; Cuzange, E.; de Lescar, R.; de Revel, G.; Sicard, G. The olfactory masking effect of ethylphenols: Characterization and elucidation of its origin. Food Q. Prefer. 2016, 50, 135-144. [CrossRef]

40. Granato, D.; Margraf, T.; Brotzakis, I.; Capuano, E.; van Ruth, S.M. Characterization of conventional, biodynamic, and organic purple grape juices by chemical markers, antioxidant capacity, and instrumental taste profile. J. Food Sci. 2015, 80, C55-C65. [CrossRef] [PubMed]

41. Spínola, V.; Perestrelo, R.; Câmara, J.S.; Castilho, P.C. Establishment of monstera deliciosa fruit volatile metabolomic profile at different ripening stages using solid-phase microextraction combined with gas chromatography-mass spectrometry. Food Res. Int. 2015, 67, 409-417. [CrossRef]

42. Ferreira, V.; Aznar, M.; López, R.; Cacho, J. Quantitative gas chromatography-olfactometry carried out at different dilutions of an extract. Key differences in the odor profiles of four high-quality Spanish aged red wines. J. Agric. Food Chem. 2001, 49, 4818-4824. [CrossRef] [PubMed]

43. Varming, C.; Petersen, M.A.; Poll, L. Comparison of isolation methods for the determination of important aroma compounds in black currant (Ribes nigrum L.) juice, using nasal impact frequency profiling. J. Agric. Food Chem. 2004, 52, 1647-1652. [CrossRef] [PubMed]

44. Paduch, R.; Kandefer-Szerszeń, M.; Trytek, M.; Fiedurek, J. Terpenes: Substances useful in human healthcare. Arch. Immunol. Ther. Exp. 2007, 55, 315-327. [CrossRef]

45. Miller, J.A.; Thompson, P.A.; Hakim, I.A.; Chow, H.-H.S.; Thomson, C.A. $d$-Limonene: A bioactive food component from citrus and evidence for a potential role in breast cancer prevention and treatment. Oncol. Rev. 2011, 5, 31-42. [CrossRef]

46. Murali, R.; Karthikeyan, A.; Saravanan, R. Protective effects of D-limonene on lipid peroxidation and antioxidant enzymes in streptozotocin-induced diabetic rats. Basic Clin. Pharmacol. Toxicol. 2013, 112, 175-181. [CrossRef] [PubMed]

47. Park, S.-N.; Lim, Y.K.; Freire, M.O.; Cho, E.; Jin, D.; Kook, J.-K. Antimicrobial Effect of Linalool and $\alpha$-Terpineol Against Periodontopathic and Cariogenic Bacteria. Anaerobe 2012, 18, 369-372. [CrossRef] [PubMed]

48. Li, Y.; Lv, O.; Zhou, F.; Li, Q.; Wu, Z.; Zheng, Y. Linalool inhibits LPS-induced inflammation in BV2 microglia cells by activating Nrf2. Neurochem. Res. 2015, 40, 1520-1525. [CrossRef] [PubMed]

49. Schmutzer, G.R.; Magdas, A.D.; David, L.I.; Moldovan, Z. Determination of the volatile components of apple juice using solid phase microextraction and gas chromatography-mass spectrometry. Anal. Lett. 2014, 47, 1683-1696. [CrossRef]

50. Perez-Cacho, P.R.; Rouseff, R.L. Fresh Squeezed Orange Juice Odor: A Review. Crit. Rev. Food Sci. Nutr. 2008, 48, 681-695. [CrossRef] [PubMed]

51. Perez-Cacho, P.R.; Rouseff, R. Processing and Storage Effects on Orange Juice Aroma: A Review. J. Agric. Food Chem. 2008, 56, 9785-9796. [CrossRef] [PubMed]

52. Arena, E.; Guarrera, N.; Campisi, S.; Nicolosi Asmundo, C. Comparison of odour active compounds detected by gas-chromatography-olfactometry between hand-squeezed juices from different orange varieties. Food Chem. 2006, 98, 59-63. [CrossRef]

53. Beaulieu, J.C.; Stein-Chisholm, R.E. HS-GC-MS volatile compounds recovered in freshly pressed 'Wonderful' cultivar and commercial pomegranate juices. Food Chem. 2016, 190, 643-656. [CrossRef] [PubMed]

54. Beaulieu, J.C.; Stein-Chisholm, R.E.; Lloyd, S.W.; Bett-Garber, K.L.; Grimm, C.C.; Watson, M.A.; Lea, J.M. Volatile, anthocyanidin, quality and sensory changes in rabbiteye blueberry from whole fruit through pilot plant juice processing. J. Sci. Food Agric. 2017, 97, 469-478. [CrossRef] [PubMed]

55. Bai, J.; Baldwin, E.; McCollum, G.; Plotto, A.; Manthey, J.; Widmer, W.; Luzio, G.; Cameron, R. Changes in volatile and non-volatile flavor chemicals of "Valencia" orange juice over the harvest seasons. Foods 2016, 5, 4. [CrossRef] [PubMed]

56. Vázquez-Araújo, L.; Koppel, K.; Chambers, E., IV; Adhikari, K.; Carbonell-Barrachina, A.A. Instrumental and sensory aroma profile of pomegranate juices from the USA: Differences between fresh and commercial juice. Flavour Fragrance J. 2011, 26, 129-138. [CrossRef] 
57. Qiao, Y.; Xie, B.J.; Zhang, Y.; Zhang, Y.; Fan, G.; Yao, X.L.; Pan, S.Y. Characterization of aroma active compounds in fruit juice and peel oil of Jinchen sweet orange fruit (Citrus sinensis (L.) Osbeck) by GC-MS and GC-O. Molecules (Basel Switzerland) 2008, 13, 1333-1344. [CrossRef]

58. Edlin, D.A.N.; Narbad, A.; Dickinson, J.R.; Lloyd, D. The biotransformation of simple phenolic compounds by Brettanomyces anomalus. FEMS Microbiol. Lett. 1995, 125, 311-315. [CrossRef]

59. Daud, M.N.H.; Ahamad, N.; Jabit, M.L.; Kormin, S. A new approach for reduction of chemical compounds causing undesirable odour in Morinda citrifolia fruit juice. J. Trop. Agric. Food Sci. 2013, 41, 257-264.

60. Perestrelo, R.; Barros, A.S.A.S.; Rocha, S.M.; Câmara, J.S. Establishment of the varietal profile of Vitis vinifera L. grape varieties from different geographical regions based on HS-SPME/GC-qMS combined with chemometric tools. Microchem. J. 2014, 116, 107-117. [CrossRef]

61. van Den Dool, H. A generalization of the retention index system including linear temperature programmed gas-Liquid partition chromatography. J. Chromatogr. A 1963, 11, 463-471. [CrossRef]

62. Acree, T.; Arn, H. Flavornet Home Page. Available online: http://www.flavornet.org/ (accessed on 14 February 2019).

63. Chong, J.; Soufan, O.; Li, C.; Caraus, I.; Li, S.; Bourque, G.; Wishart, D.S.; Xia, J. MetaboAnalyst 4.0: Towards more transparent and integrative metabolomics analysis. Nucleic Acids Res. 2018, 46, W486-W494. [CrossRef] [PubMed]

Sample Availability: Samples of the compounds are not available from the authors. 